九州大学学術情報リポジトリ

Kyushu University Institutional Repository

\title{
Wave radiation from multiple cylinders of arbitrary cross sections
}

Zheng, Siming

School of Engineering, University of Plymouth

Zhang, Yongliang

State Key Laboratory of Hydroscience and Engineering, Tsinghua University

Liu, Yingyi

Research Institute for Applied Mechanics, Kyushu University

Iglesias, Gregorio

School of Engineering, University of Plymouth

http://hdl. hand le. net/2324/4055221

出版情報：Ocean Engineering. 184，pp.11-22，2019-07-15. Elsevier バージョン：

権利関係: Creative Commons Attribution NonCommercial NoDerivatives 4.0 International 
1 Title:

2 Wave radiation from multiple cylinders of arbitrary cross sections

3

4 Author names and affiliations:

5 Siming Zheng ${ }^{\mathrm{a}, \mathrm{b}}$, Yongliang Zhang ${ }^{\mathrm{b}}$, Yingyi Liu ${ }^{\mathrm{c}}$, Gregorio Iglesias ${ }^{\mathrm{d}, \mathrm{a}}$

6

7 a. School of Engineering, University of Plymouth, Drake Circus, Plymouth PL4 8AA, UK

8 b. State Key Laboratory of Hydroscience and Engineering, Tsinghua University, Beijing 100084,

9 China

10 c. Research Institute for Applied Mechanics, Kyushu University, Kasuga, Fukuoka 816-8580,

11 Japan

12 d. MaREI, Environmental Research Institute \& School of Engineering, University College Cork,

13 Western Road, Cork, Ireland

14

E-mail addresses:

16 Siming Zheng ${ }^{\mathrm{a}, \mathrm{b}}$ siming.zheng@plymouth.ac.uk/zhengsm@tsinghua.edu.cn

17 Yongliang Zhang ${ }^{\mathrm{b}}$ yongliangzhang@tsinghua.edu.cn

18 Yingyi Liu ${ }^{\mathrm{c}}$ liuyingyi@riam.kyushu-u.ac.jp

19 Gregorio Iglesias ${ }^{\mathrm{d}, \mathrm{a}}$ gregorio.iglesias@ucc.ie

20

21

22

23

24

25

26

\section{Corresponding author:}

Gregorio Iglesias

Tel: +353214902523

E-mail address: gregorio.iglesias@ucc.ie

https://doi.org/10.1016/j.oceaneng.2019.05.018

Received 15 January 2019,

Revised 1 May 2019,

Accepted 8 May 2019,

Available online 17 May 2019. 


\title{
Wave radiation from multiple cylinders of arbitrary cross sections
}

\begin{abstract}
A semi-analytic model based on the linear potential theory is developed to solve wave radiation from an array of truncated cylinders with arbitrary cross sections, which are free to oscillate independently in the water of finite depth. The radiated velocity potential in the interior region below each cylinder and an exterior region extending to the infinity are expressed as a series of eigenfunctions in the local cylindrical coordinate system using the method of separation of variables. They are solved subsequently by imposing the continuity condition of the velocity and the pressure at the wetted surfaces of the cylinders and the fluid interfaces between adjacent regions. In the process, the Fourier series expansion method is applied to the terms regarding the arbitrary cross sections. The model is successfully validated through a case study with two caissons, and an excellent agreement is obtained with a numerical model based on the boundary element method. The semi-analytic model is then applied to explore the influence of the layout (angle and spacing between the two caissons) on the added-mass and radiation damping coefficients. It is found that the hydrodynamic interaction between the two caissons can increase or decrease the value of the coefficients depending on the wave frequency.
\end{abstract}

Keywords: Wave radiation; Floating cylinder; Hydrodynamic coefficients; Added mass; Radiation damping; Potential flow.

\section{Introduction}

Floating structures undergoing harmonic oscillations in still water produce radiated waves; the oscillations cause the water to react on the structure, resulting in hydrodynamic forces - the socalled wave radiation forces. It is well known that the response of marine structures can be significantly influenced by the radiation forces. Therefore, solving the problem of wave radiation from offshore structures has been a subject of considerable research interest (Bai and Eatock Taylor, 2006; Krishnankutty and Vendhan, 1995; Newman, 2005; Sheng et al., 2015; Taghipour et al., 2008).

The radiation forces exerted on structures oscillating harmonically are generally split into two terms: one, in phase with the velocity of the structure; the other, with its acceleration. Hydrodynamic coefficients - more specifically, radiation damping and added-masses - are introduced to express the radiation forces. To solve the radiation problem of a truncated circular cylinder oscillating timeharmonically in finite-depth water, Yeung (1981) divided the water domain into an interior region below the cylinder and an exterior region extending to the infinity in the horizontal plane. The velocity potential in each region was expressed as a series of eigenfunctions in a cylindrical coordinate system. The hydrodynamic coefficients, including coupling coefficients for sway and roll, were obtained analytically. A similar analytical method was applied by Bhatta and Rahman (2003) and Bhatta (2007) to compute the added-mass and damping coefficients in the case of a heaving circular cylinder, for various depth-to-radius and draft-to-radius ratios. For a given depthto-radius ratio, the heave added-mass was found to increase with the draft-to-radius ratio. Jiang et al. (2014) proposed analytical solutions for the wave radiation by a submerged circular cylinder, and found that the heave and pitch added-masses of the cylinder can be twice as large as that of itself. Circular cylinders in other situations, e.g., floating in extremely shallow water or in front of 
a vertical wall, have also been studied (Drobyshevski, 2004; Teng et al., 2004; Yu and Srivastava, 2012; Zheng and Zhang, 2015, 2016).

Wave radiation from an elliptical cylinder either submerged and resting on the seabed or partially immersed was analytically investigated by Williams and Darwiche (1990). As in Yeung (1981), the fluid was divided into interior and exterior regions; in contrast, the governing equation and boundary conditions were written in elliptical coordinates, and the velocity potentials in each region were expressed in terms of infinite series involving Mathieu and modified Mathieu functions. This technique was later adopted by Zhang and Williams (1996) in solving the wave radiation problem from a submerged elliptical disk. Hydrodynamic properties of the disk were found to be strongly dependent on both the aspect ratio and relative submergence. More recently, Yu et al. (2019) proposed an analytical model that can be used to deal with the problem of radiation from a floating cylinder with an arbitrary cross section. The Fourier series method was adopted to describe the wetted surface body conditions and continuity conditions between adjacent regions.

In the case of an array of cylinders - ubiquitous in ocean energy and offshore engineering applications, e.g., offshore wind farms and wave farms - the oscillation of the cylinders in unison or independently from one another will give rise to hydrodynamic interactions between them, further complicating the radiation problem. Kagemoto and Yue (1986) developed an interaction theory which can be used to predict the wave hydrodynamic coefficients given only the diffraction characteristics of individual members. Following Linton and Evans (1990), who had applied a direct method to the wave diffraction at multiple circular uniform cylinders, Kim (1993) studied the wave radiation for six-degree-of-freedom motions of $N$ bottom-mounted vertical circular cylinders and obtained the radiation potentials as well as the added-mass and wave damping coefficients in closed forms. Yilmaz and Incecik (1998) employed Kagemoto and Yue (1986)'s method to calculate the hydrodynamic coefficients of an array of truncated circular cylinders oscillating as a whole. Therefore, his model cannot be used to solve the radiation problem of an array of cylinders if they oscillate individually, as is the case in a floating offshore wind farm or a wave farm. To overcome this limitation, Siddorn and Eatock Taylor (2008) considered wave diffraction and radiation by multiple truncated circular cylinders which are free to oscillate independently. Extensive results were presented for the hydrodynamic coefficients of a group of four truncated circular cylinders free to respond independently in translational modes, i.e., surge, sway and heave. The analytical results of the hydrodynamic coefficients in rotational modes, e.g., roll and pitch, can be found in Zeng and Tang (2013). More recently, Zheng and Zhang (2018) and Zheng et al. (2018) investigated the wave radiation from an array of truncated circular cylinders with one or some of them hollowed out. Concerning non-circular cylinders, the hydrodynamic problems of arrays of elliptical cylinders subjected to linear regular waves were studied analytically by Chatjigeorgiou and Mavrakos (2010) and Chatjigeorgiou (2013), considering fixed cylinders, therefore with no wave radiation forces.

The objective of this paper is to investigate wave radiation from multiple truncated cylinders with arbitrary cross sections and free to oscillate independently from one another, considering the hydrodynamic interactions between them - a problem that has not been addressed so far. This is carried out by means of an ad hoc semi-analytic model, which is used to evaluate the added-mass and wave radiation damping coefficients, together with the hydrodynamic interaction coefficients. The semi-analytic model constitutes an option to deal with wave radiation from multiple vertical cylinders with arbitrary cross sections without the utilization of any BEM-based numerical software. 


\section{Mathematical model}

An array of truncated cylinders of arbitrary cross sections floating in water with a depth of $h$ (Fig. 1) is considered. The cylinders are numbered $n=1,2, \ldots, N$, and located in a Cartesian coordinate system $O x y z$ with the plane $z=0$ on the mean water level (MWL) and the $O z$ axis pointing vertically upwards. For Cylinder $n$, a cylindrical coordinate system denoted as $O_{n} r_{n} \theta_{n} z$ is adopted, with the origin $O_{n}$ set within the cross sections of the cylinder at MWL, with coordinates $\left(x_{n}, y_{n}, 0\right)$ in the $O x y z$ system. A point defined as $\left(x_{n}, y_{n}, z_{n}\right)$ in the $O x y z$ system is adopted as the reference point of the rotational modes of Cylinder $n$ to consider the radiation problem induced by the rotational motions. The submerged depth of each cylinder can be different, denoted as $d_{n}$ for Cylinder $n$, respectively.
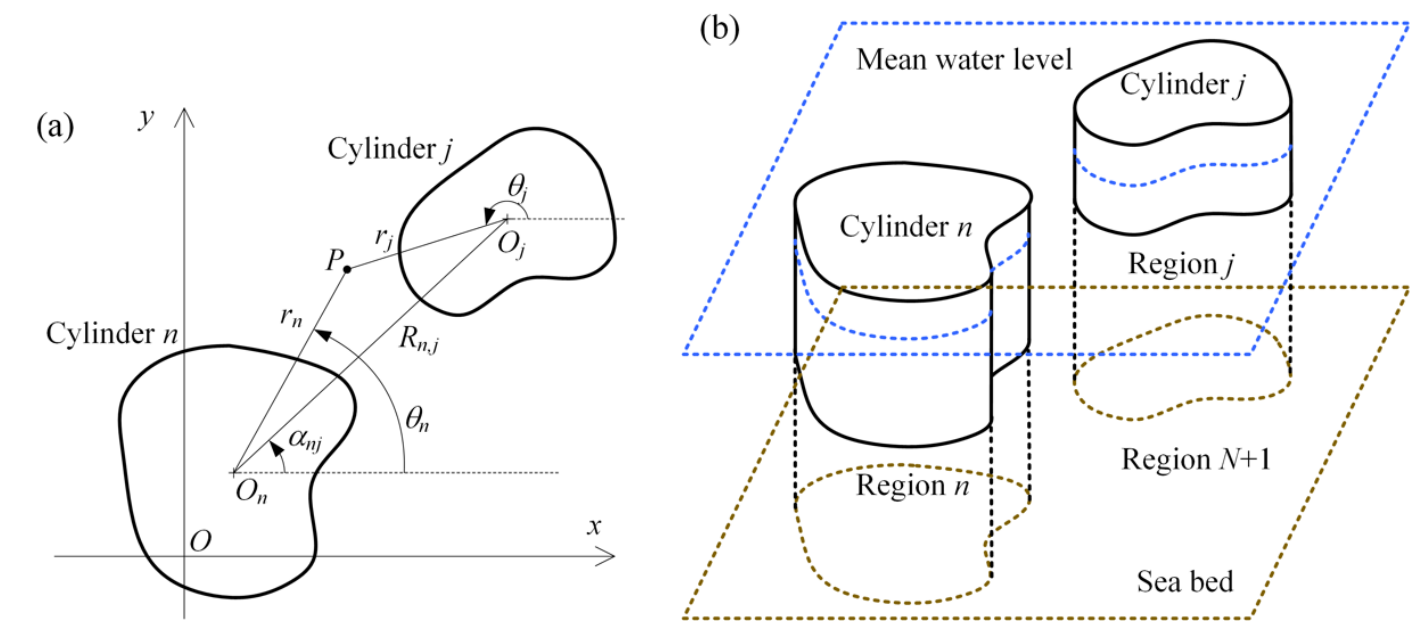

Fig. 1. Schematic of the truncated cylinders of arbitrary cross sections: a) plan view; b). side view

To describe the shape of the cross section of Cylinder $n$, a function denoted as $S_{n}$ is introduced in the $O_{n} r_{n} \theta_{n} z$ system as:

$$
S_{n}\left(r_{n}, \theta_{n}\right)=r_{n}-R_{n}\left(\theta_{n}\right),
$$

where $R_{n}\left(\theta_{n}\right)$ denotes the radius of any point at the edge of the cross section at $\theta_{n}$. Therefore, the normal unit vector pointing into the water at the side surface of Cylinder $n$ can be written in the $O_{n} r_{n} \theta_{n} z$ system as:

$$
\vec{n}=\frac{1}{\sqrt{1+\left(\frac{1}{r_{n}} \frac{\partial S_{n}}{\partial \theta_{n}}\right)^{2}}}\left(\vec{e}_{r, n}+\frac{1}{r_{n}} \frac{\partial S_{n}}{\partial \theta_{n}} \vec{e}_{\theta, n}+0 \vec{e}_{z}\right),
$$

in which $\left(\vec{e}_{r, n}, \vec{e}_{\theta, n}, \vec{e}_{z}\right)$ are the unit basis vectors in the $O_{n} r_{n} \theta_{n} z$ system.

For a cylinder with the cross-section edge varying sharply at some points, as in the case of rectangular and triangular cylinders, the normal unit vector at the side surface might be discontinuous at these points. For such cases, a series of Fourier functions can be adopted to represent $R_{n}$ and $S_{n}$, following Liu et al. (2017).

As shown in Fig. 1b, the entire water domain is divided into $N$ interior regions in which Region $n(n=1,2, \ldots, N)$ is the "projection" region below Cylinder $n$, bounded by the cylinder bottom, seabed and the extension of the cylinder side surface, and a single exterior region, i.e., 
Region $N+1$, which circumscribes the cylinders and their "projections" and extends to the horizontal infinity.

It is assumed that water is inviscid and incompressible, the flow is irrotational, and each of the cylinders oscillates harmonically with a small amplitude; therefore, the linear potential theory may be used to investigate the radiation problem from multiple cylinders. When the multiple cylinders oscillate, radiated waves are generated, and the total radiated velocity potential can be expressed as

$$
\phi(x, y, z, t)=\operatorname{Re}\left[\Phi(x, y, z) \mathrm{e}^{-\mathrm{i} \omega t}\right],
$$

where $t$ is the time, $\Phi$ is a complex spatial velocity potential independent of time, $\mathrm{i}=\sqrt{-1}$, and $\omega$ is the angular frequency of the harmonic oscillation.

The spatial velocity potential $\Phi$ may be expressed as a superposition (linear combination) of radiated potentials caused by each individual cylinder oscillating in six modes of oscillation:

$$
\Phi=\sum_{n=1}^{N} \sum_{i=1}^{6} \Phi_{\mathrm{R}}^{n, i} u^{n, i}
$$

where $\Phi_{\mathrm{R}}^{n, i}$ represents the spatial velocity potential due to a unit-amplitude velocity oscillation of Cylinder $n$ in $i$-th mode ( $i=1,2 \ldots 6$ denoting the modes of surge, sway, heave, roll, pitch, and yaw, respectively), and $u^{n, i}$ denotes the corresponding complex velocity amplitude.

It follows that $\Phi_{\mathrm{R}}^{n, i}$ must satisfy the three-dimensional Laplace's equation in the entire water domain,

$$
\frac{\partial^{2} \Phi_{\mathrm{R}}^{n, i}}{\partial x^{2}}+\frac{\partial^{2} \Phi_{\mathrm{R}}^{n, i}}{\partial y^{2}}+\frac{\partial^{2} \Phi_{\mathrm{R}}^{n, i}}{\partial z^{2}}=0
$$

the kinematical condition on the seabed,

$$
\frac{\partial \Phi_{\mathrm{R}}^{n, i}}{\partial z}=0, \quad z=-h,
$$

should also be satisfied, as well as the linearized free surface condition,

$$
\frac{\partial \Phi_{\mathrm{R}}^{n, i}}{\partial z}-\frac{\omega^{2}}{g} \Phi_{\mathrm{R}}^{n, i}=0, \quad z=0 \text { and } S_{j} \geq 0 .
$$

The boundary conditions at the wetted surface of all cylinders that $\Phi_{R}^{n, i}$ must satisfy can be written as:

$$
\frac{\partial \Phi_{\mathrm{R}}^{n, i}}{\partial z}=\delta_{n, j}\left(\delta_{3, i}+\delta_{4, i} r_{j} \sin \theta_{j}-\delta_{5, i} r_{j} \cos \theta_{j}\right), \quad z=-d_{j} \text { and } S_{j}<0
$$

$$
r_{j}^{2} \frac{\partial \Phi_{\mathrm{R}}^{n, i}}{\partial r_{j}}+\frac{\partial S_{j}}{\partial \theta_{j}} \frac{\partial \Phi_{\mathrm{R}}^{n, i}}{\partial \theta_{j}}=\delta_{n, j} f_{1}^{n, i}, \quad-d_{j} \leq z \leq 0 \quad \text { and } \quad S_{j}=0
$$

where $\delta_{i, j}$ represents the Kronecker delta function, and 


$$
f_{1}^{n, i}= \begin{cases}r_{n}^{2}\left(\cos \theta_{n}-\frac{\partial S_{n}}{r_{n} \partial \theta_{n}} \sin \theta_{n}\right), & i=1 \\ r_{n}^{2}\left(\sin \theta_{n}+\frac{\partial S_{n}}{r_{n} \partial \theta_{n}} \cos \theta_{n}\right), & i=2 \\ -\left(z-z_{n}\right) r_{j}^{2}\left(\sin \theta_{n}+\frac{\partial S_{n}}{r_{n} \partial \theta_{n}} \cos \theta_{n}\right), & i=4 \\ \left(z-z_{n}\right) r_{n}^{2}\left(\cos \theta_{n}-\frac{\partial S_{n}}{r_{n} \partial \theta_{n}} \sin \theta_{n}\right), & i=5 \\ r_{n}^{2} \frac{\partial S_{n}}{\partial \theta_{n}}, & i=6\end{cases}
$$

The radiation condition at infinity is:

$$
\sqrt{k_{0} r_{n}}\left(\frac{\partial \Phi_{\mathrm{R}}^{n, i}}{\partial r_{n}}-\mathrm{i} k_{0} \Phi_{\mathrm{R}}^{n, i}\right)=0, \quad S_{j} \rightarrow \infty
$$

where $k_{0}$ is the wave number, which satisfies the dispersion relation, i.e.,

$$
\omega^{2}=g k_{0} \tanh \left(k_{0} h\right) .
$$

\section{The solution to radiated potentials}

\subsection{Radiated spatial potentials in different regions}

For interior and exterior regions, i.e., Region $j(j=1,2, \ldots, N)$ and Region $N+1$, as divided in Fig. 1b, the radiated spatial potential can be expressed in different forms. The spatial velocity potentials $\Phi_{\mathrm{R}}^{n, i}$ in Region $j$, denoted as $\Phi_{\mathrm{R}, j}^{n, i}$, are given as follows:

1) Region $j(j=1,2, \ldots, N)$

$$
\Phi_{\mathrm{R}, j}^{n, i}\left(r_{j}, \theta_{j}, z\right)=\delta_{n, j} \Phi_{\mathrm{R}, \mathrm{p}}^{n, i}+\sum_{m=-\infty}^{\infty}\left[A_{m, 0}^{n, i, j}\left(r_{j}\right)^{|m|}+\sum_{l=1}^{\infty} A_{m, l}^{n, i, j} I_{m}\left(\beta_{j, l} r_{j}\right) \cos \left[\beta_{j, l}(z+h)\right]\right] \mathrm{e}^{\mathrm{i} m \theta_{j}},
$$

where $A_{m, l}^{n, i, j}$ are the unknown coefficients to be determined; $I_{m}$ is the modified Bessel function of first kind and order $m ; \beta_{j, l}$ is the eigenvalue, which is given by

$$
\beta_{j, l}=\frac{l \pi}{h-d_{j}}, \quad l=0,1,2,3, \ldots
$$

\footnotetext{
$\Phi_{\mathrm{R}, \mathrm{p}}^{n, i}$ is a particular solution, which can be expressed in the $O_{n} r_{n} \theta_{n} z$ coordinate system as
} 


$$
\Phi_{\mathrm{R}, \mathrm{p}}^{n, i}=\left\{\begin{array}{ll}
0, \quad i=1,2,6 \\
\frac{1}{4\left(h-d_{n}\right)}\left[2(z+h)^{2}-r_{n}^{2}\right], & i=3 \\
\frac{\sin \theta_{n}}{8\left(h-d_{n}\right)}\left[4 r_{n}(z+h)^{2}-r_{n}^{3}\right], & i=4 \\
\frac{\cos \theta_{n}}{8\left(h-d_{n}\right)}\left[r_{n}^{3}-4 r_{n}(z+h)^{2}\right], & i=5
\end{array} .\right.
$$

\section{2) Region $N+1$}

In Region $N+1$, the radiated spatial potential can be decomposed into the summation of $N$ cylindrical spatial potentials radiated from $N$ cylinders:

$$
\Phi_{\mathrm{R}, N+1}^{n, i}=\sum_{j=1}^{N} \Phi_{j}^{n, i, \mathrm{e}},
$$

where $\Phi_{j}^{n, i, \mathrm{e}}$ represents the wave traveling outwards from Cylinder $j$, which can be written in terms of the following well-known eigenfunction expansion in its cylindrical coordinate system:

$$
\Phi_{j}^{n, i, \mathrm{e}}\left(r_{j}, \theta_{j}, z\right)=\sum_{m=-\infty}^{\infty}\left[B_{m, 0}^{n, i, j} H_{m}\left(k_{0} r_{j}\right) \frac{Z_{0}(z)}{Z_{0}(0)}+\sum_{l=1}^{\infty} B_{m, l}^{n, i, j} K_{m}\left(k_{l} r_{j}\right) \frac{Z_{l}(z)}{Z_{l}(0)}\right] \mathrm{e}^{\mathrm{i} m \theta_{j}}
$$

in which

$$
\begin{aligned}
& Z_{0}(z)=N_{0}^{-1 / 2} \cosh \left[k_{0}(z+h)\right] ; \quad Z_{l}(z)=N_{l}^{-1 / 2} \cos \left[k_{l}(z+h)\right] \\
& N_{0}=\frac{1}{2}\left[1+\frac{\sinh \left(2 k_{0} h\right)}{2 k_{0} h}\right] ; \quad N_{l}=\frac{1}{2}\left[1+\frac{\sin \left(2 k_{l} h\right)}{2 k_{l} h}\right] ;
\end{aligned}
$$

$B_{m, 0}^{n, i, j}$ are the coefficients to be solved in Section 3.2; $H_{m}$ is the Hankel function of the first kind of order $m ; K_{m}$ is the modified Bessel function of the second kind of order $m ; k_{l}$ is the eigenvalue, which is given by

$$
\omega^{2}=-k_{l} g \tan \left(k_{l} h\right), \quad l=1,2,3, \ldots
$$

By using the Graf's addition theorem for Bessel functions (Abramowitz and Stegun, 1964; Zheng and Zhang, 2015, 2016, 2018), the expression of $\Phi_{n^{\prime}}^{n, i, \mathrm{e}}$ can be converted from its own $\mathrm{O}_{n} \cdot r_{n} \cdot \theta_{n} \cdot z$ system into the $\mathrm{O}_{j} r_{j} \theta_{j} z$ system $\left(n^{\prime} \neq j\right)$ for $r_{j} \leq R_{j n^{\prime}}$, and $\Phi_{\mathrm{R}, N+1}^{n, i}$ in the $\mathrm{O}_{j} r_{j} \theta_{j} z$ system can be expressed by:

$$
\begin{aligned}
\Phi_{\mathrm{R}, N+1}^{n, i}\left(r_{j}, \theta_{j}, z\right) & =\sum_{m=-\infty}^{\infty}\left[B_{m, 0}^{n, i, j} H_{m}\left(k_{0} r_{j}\right) \frac{Z_{0}(z)}{Z_{0}(0)}+\sum_{l=1}^{\infty} B_{m, l}^{n, i, j} K_{m}\left(k_{l} r_{j}\right) \frac{Z_{l}(z)}{Z_{l}(0)}\right] \mathrm{e}^{\mathrm{i} m \theta_{j}} \\
& +\sum_{\substack{n^{\prime}=1 \\
n^{\prime \neq j}}}^{N} \sum_{m=-\infty}^{\infty}\left\{B_{m, 0}^{n, i, n^{\prime}} \frac{Z_{0}(z)}{Z_{0}(0)} \sum_{m^{\prime}=-\infty}^{\infty}(-1)^{m^{\prime}} H_{m-m^{\prime}}\left(k_{0} R_{n^{\prime} j}\right) J_{m^{\prime}}\left(k_{0} r_{j}\right) \mathrm{e}^{\mathrm{i}\left(m \alpha_{n j^{\prime}}-m^{\prime} \alpha_{j n^{\prime}}\right.}\right) \mathrm{e}^{\mathrm{i} m^{\prime} \theta_{j}}, \\
& \left.+\sum_{l=1}^{\infty} B_{m, l}^{n, i, n^{\prime}} \frac{Z_{l}(z)}{Z_{l}(0)} \sum_{m^{\prime}=-\infty}^{\infty} K_{m-m^{\prime}}\left(k_{l} R_{n^{\prime} j}\right) I_{m^{\prime}}\left(k_{l} r_{j}\right) \mathrm{e}^{\mathrm{i}\left(m \alpha_{n j^{\prime}}-m^{\prime} \alpha_{j n^{\prime}}\right)} \mathrm{e}^{\mathrm{i} m^{\prime} \theta_{j}}\right\}
\end{aligned}
$$

where $J_{m}$ is the Bessel function of order $m$. 


\subsection{Computation method for the coefficients}

Eqs. (13) and (21) for the radiated spatial potentials given in Sections 3.1 must also satisfy the boundary conditions, Eq. (9), and the continuity conditions on the interfaces of any two adjacent regions, i.e., $S_{j}=0(j=1,2, \ldots, N)$. These conditions can be combined as follows:

1) Continuity of pressure at the boundary $S_{j}=0$ :

$$
\left.\Phi_{\mathrm{R}, N+1}^{n, i}\right|_{S_{j}=0}=\left.\Phi_{\mathrm{R}, j}^{n, i}\right|_{S_{j}=0}, \quad-h<z<-d_{j},
$$

2) Continuity of normal velocity at the boundary $S_{j}=0$ :

$$
\left.\left(r_{j}^{2} \frac{\partial_{\mathrm{R}, N+1}^{n, i}}{\partial r_{j}}+\frac{\partial S_{j}}{\partial \theta_{j}} \frac{\partial_{\mathrm{R}, N+1}^{n, i}}{\partial \theta_{j}}\right)\right|_{S_{j}=0}=\left\{\begin{array}{l}
\left.\delta_{n, j} f_{1}^{n, i}\right|_{S_{j}=0}, \quad-d_{j}<z<0 \\
\left.\left(r_{j}^{2} \frac{\partial \Phi_{\mathrm{R}, j}^{n, i}}{\partial r_{j}}+\frac{\partial S_{j}}{\partial \theta_{j}} \frac{\partial \Phi_{\mathrm{R}, j}^{n, i}}{\partial \theta_{j}}\right)\right|_{S_{j}=0},-h<z<-d_{j}
\end{array}\right.
$$

After inserting Eqs. (13) and (21) into Eqs. (22) and (23), it is readily observed that the terms obtained regarding $r_{n}$ and $\partial S_{n} / \partial \theta_{n}$ are both dependent on $\theta_{n}$ at $S_{n}=0$. Fourier series can be employed to express these items as follows:

$$
\left.J_{m}\left(k_{0} r_{n}\right)\right|_{S_{n}=0}=\sum_{q=-\infty}^{\infty} f_{m, 0, q}^{J, n} \mathrm{e}^{\mathrm{i} q \theta_{n}} ;\left.\left(r_{n}^{2} k_{0} J_{m}^{\prime}\left(k_{0} r_{n}\right)+\mathrm{i} m J_{m}\left(k_{0} r_{n}\right) \frac{\partial S_{n}}{\partial \theta_{n}}\right)\right|_{S_{n}=0}=\sum_{q=-\infty}^{\infty} f_{m, 0, q}^{J^{\prime}, n} \mathrm{e}^{\mathrm{i} q \theta_{n}},
$$

$\left.H_{m}\left(k_{0} r_{n}\right)\right|_{S_{n}=0}=\sum_{q=-\infty}^{\infty} f_{m, 0, q}^{H, n} \mathrm{e}^{\mathrm{i} q \theta_{n}} ;\left.\left(r_{n}^{2} k_{0} H_{m}^{\prime}\left(k_{0} r_{n}\right)+\mathrm{i} m H_{m}\left(k_{0} r_{n}\right) \frac{\partial S_{n}}{\partial \theta_{n}}\right)\right|_{S_{n}=0}=\sum_{q=-\infty}^{\infty} f_{m, 0, q}^{H^{\prime}, n} \mathrm{e}^{\mathrm{i} q \theta_{n}}$

$$
\left.K_{m}\left(k_{l} r_{n}\right)\right|_{S_{n}=0}=\sum_{q=-\infty}^{\infty} f_{m, l, q}^{K, n} \mathrm{e}^{\mathrm{i} q \theta_{n}} ;\left.\left(r_{n}^{2} k_{l} K_{m}^{\prime}\left(k_{l} r_{n}\right)+\mathrm{i} m K_{m}\left(k_{l} r_{n}\right) \frac{\partial S_{n}}{\partial \theta_{n}}\right)\right|_{S_{n}=0}=\sum_{q=-\infty}^{\infty} f_{m, l, q}^{K^{\prime}, n} \mathrm{e}^{\mathrm{i} q \theta_{n}}
$$

$\left.I_{m}\left(k_{l} r_{n}\right)\right|_{S_{n}=0}=\sum_{q=-\infty}^{\infty} f_{m, l, q}^{I, n} \mathrm{e}^{\mathrm{i} q \theta_{n}} ;\left.\left(r_{n}^{2} k_{l} I_{m}^{\prime}\left(k_{l} r_{n}\right)+\mathrm{i} m I_{m}\left(k_{l} r_{n}\right) \frac{\partial S_{n}}{\partial \theta_{n}}\right)\right|_{S_{n}=0}=\sum_{q=-\infty}^{\infty} f_{m, l, q}^{I^{\prime}, n} \mathrm{e}^{\mathrm{i} q \theta_{n}}$

$$
\left.I_{m}\left(\beta_{n, l} r_{n}\right)\right|_{S_{n}=0}=\sum_{q=-\infty}^{\infty} \tilde{f}_{m, l, q}^{I, n} \mathrm{e}^{\mathrm{i} q \theta_{n}} ;\left.\left(r_{n}^{2} \beta_{n, l} I_{m}^{\prime}\left(\beta_{n, l} r_{n}\right)+\mathrm{i} m I_{m}\left(\beta_{n, l} r_{n}\right) \frac{\partial S_{n}}{\partial \theta_{n}}\right)\right|_{S_{n}=0}=\sum_{q=-\infty}^{\infty} \tilde{f}_{m, l, q}^{I^{\prime}, n} \mathrm{e}^{\mathrm{i} q \theta_{n}}
$$

$$
\left.\left(r_{n}\right)^{|m|}\right|_{S_{n}=0}=\sum_{q=-\infty}^{\infty} f_{m, 0, q}^{R, n} \mathrm{e}^{\mathrm{i} q \theta_{n}} ;\left.\quad\left(|m|\left(r_{n}\right)^{|m|+1}+\mathrm{i} m\left(r_{n}\right)^{|m|} \frac{\partial S_{n}}{\partial \theta_{n}}\right)\right|_{S_{n}=0}=\sum_{q=-\infty}^{\infty} \tilde{f}_{m, 0, q}^{R, n} \mathrm{e}^{\mathrm{i} q \theta_{n}},
$$

in which, for convenience, the Fourier coefficients on the right-hand side of Eqs. (24a) (24f) can be represented by $\mathfrak{R}_{n, q}$ and evaluated from

$$
\mathfrak{R}_{n, q}=\frac{1}{2 \pi} \int_{-\pi}^{\pi} \Psi_{n, q}\left(\theta_{n}\right) \mathrm{e}^{-\mathrm{i} q \theta_{n}} \mathrm{~d} \theta_{n},
$$

where $\Psi_{n, q}$ denotes the $\theta_{n}$ dependent items as given on the left-hand side of Eqs. (24a) $\sim(24 \mathrm{f})$.

Multiplying both sides of Eq. (22) by $\mathrm{e}^{-\mathrm{i} z \theta_{j}} \cos \left[\beta_{j, \xi}(z+h)\right] /\left(h-d_{j}\right)$, integrating for $\theta_{j} \in[-\pi, \pi]$ and $z \in\left[-h,-d_{j}\right]$, and making some rearrangements, we obtain, for any pair of integer $(\tau, \xi)$ : 


$$
-\sum_{m=-\infty}^{\infty} \Lambda_{m, \tau, \xi}^{1, j} A_{m, \xi}^{n, i, j}+\sum_{m=-\infty}^{\infty} \sum_{l=0}^{\infty} B_{m, l}^{n, i, j} L_{l, \xi}^{(j)} \Lambda_{m, \tau, l}^{2, j}+\sum_{\substack{n^{\prime}=1 \\ n^{\prime} \neq j}}^{N} \sum_{m=-\infty}^{\infty} \sum_{l=0}^{\infty} B_{m, l}^{n, i, n^{\prime}} L_{l, \xi}^{(j)} T_{m, \tau, l}^{j, n^{\prime}}=\delta_{n, j} f_{\tau, \xi}^{(1), n, i},
$$

2

where

3

$$
\begin{aligned}
& \Lambda_{m, \tau, \xi}^{1, n}=\left\{\begin{array}{l}
f_{m, \xi, \tau-m}^{R, n}, \quad \xi=0 \\
\frac{1}{2} \tilde{f}_{m, \xi, \tau-m}^{I, n}, \quad \xi=1,2,3, \ldots
\end{array}, \Lambda_{m, \tau, l}^{2, n}=\left\{\begin{array}{ll}
f_{m, 0, \tau-m}^{H, n}, & l=0 \\
f_{m, l, \tau-m}^{K, n}, & l=1,2,3, \ldots
\end{array},\right.\right. \\
& L_{l, \xi}^{(n)}=\frac{1}{h-d_{n}} \int_{-h}^{-d_{n}} \frac{Z_{l}(z) \cos \left[\beta_{n, \xi}(z+h)\right]}{Z_{l}(0)} \mathrm{d} z \\
& =\left\{\begin{array}{ll}
\frac{(-1)^{\xi}\left(h-d_{n}\right) k_{0} \sinh \left[k_{0}\left(h-d_{n}\right)\right]}{\left[\left(h-d_{n}\right)^{2} k_{0}^{2}+\xi^{2} \pi^{2}\right] \cosh \left(k_{0} h\right)} & l=0 ; \xi=0,1,2 \cdots, \\
\frac{(-1)^{\xi}\left(h-d_{n}\right) k_{l} \sin \left[k_{l}\left(h-d_{n}\right)\right]}{\left[\left(h-d_{n}\right)^{2} k_{l}^{2}-\xi^{2} \pi^{2}\right] \cos \left(k_{l} h\right)} & l=1,2,3 \cdots ; \xi=0,1,2 \cdots
\end{array},\right. \\
& T_{m, \tau, l}^{n, j}=\left\{\begin{array}{ll}
\sum_{m^{\prime}=-\infty}^{\infty}(-1)^{m^{\prime}} H_{m-m^{\prime}}\left(k_{0} R_{j n}\right) f_{m^{\prime}, 0, \tau-m^{\prime}}^{J, n} \mathrm{e}^{\mathrm{i}\left(m \alpha_{j n}-m^{\prime} \alpha_{n j}\right)}, & l=0 \\
\sum_{m^{\prime}=-\infty}^{\infty} K_{m-m^{\prime}}\left(k_{l} R_{j n}\right) f_{m^{\prime}, l, \tau-m^{\prime}}^{I, n} \mathrm{e}^{\mathrm{i}\left(m \alpha_{j n}-m^{\prime} \alpha_{n j}\right)}, & l=1,2,3, \ldots
\end{array},\right. \\
& f_{\tau, \xi}^{(1), n, i}=\left\{\begin{array}{l}
0, \quad i=1,2,6 \\
\frac{1}{4\left(h-d_{n}\right)^{2}}\left[2 \delta_{\tau, 0} I Z_{n, \xi}-\delta_{\xi, 0}\left(h-d_{n}\right) f_{2,0, \tau}^{R, n}\right], \quad i=3 \\
\frac{1}{16 \mathrm{i}\left(h-d_{n}\right)^{2}}\left[4 I Z_{n, \xi}\left(f_{1,0, \tau-1}^{R, n}-f_{1,0, \tau+1}^{R, n}\right)-\delta_{\xi, 0}\left(h-d_{n}\right)\left(f_{3,0, \tau-1}^{R, n}-f_{3,0, \tau+1}^{R, n}\right)\right], \quad i=4 \\
\frac{-1}{16\left(h-d_{n}\right)^{2}}\left[4 I Z_{n, \xi}\left(f_{1,0, \tau-1}^{R, n}+f_{1,0, \tau+1}^{R, n}\right)-\delta_{\xi, 0}\left(h-d_{n}\right)\left(f_{3,0, \tau-1}^{R, n}+f_{3,0, \tau+1}^{R, n}\right)\right], \quad i=5
\end{array}\right.
\end{aligned}
$$

7

in which

8

$$
I Z_{n, \xi}=\int_{-h}^{d_{n}} \cos \left[\beta_{n, \xi}(z+h)\right](z+h)^{2} \mathrm{~d} z= \begin{cases}\frac{\left(h-d_{n}\right)^{3}}{3}, & \xi=0 \\ \frac{2(-1)^{\xi}\left(h-d_{n}\right)^{3}}{\xi^{2} \pi^{2}}, & \xi=1,2,3, \ldots\end{cases}
$$

9

After multiplying both sides of Eq.(23) by $\mathrm{e}^{-\mathrm{i} z \theta_{j}} Z_{\xi}(z) / h$ and integrating for $\theta_{j} \in[-\pi, \pi]$ and $z$ $\in[-h, 0]$, and making some rearrangements, we obtain, for any pair of integer $(\tau, \xi)$ :

$$
-\frac{h-d_{j}}{h} Z_{\xi}(0) \sum_{m=-\infty}^{\infty} \sum_{l=0}^{\infty} A_{m, l}^{n, i, j} L_{\xi, l}^{(j)} \Lambda_{m, \tau, l}^{\beta, j}+\sum_{m=-\infty}^{\infty} B_{m, \xi}^{n, i, j} \Lambda_{m, \tau, \xi}^{4, j}+\sum_{\substack{n^{\prime}=1 \\ n^{\prime} \neq j}}^{N} \sum_{m=-\infty}^{\infty} B_{m, \xi}^{n, i, n^{\prime}} T_{m, \tau, \xi}^{j, n^{\prime}}=\delta_{n, j} f_{\tau, \xi}^{(2), n, i},
$$

where 


$$
\begin{gathered}
\Lambda_{m, \tau, l}^{3, n}=\left\{\begin{array}{ll}
\tilde{f}_{m, 0, \tau-m}^{R, n}, & l=0 \\
\tilde{f}_{m, l, \tau-m}^{I^{\prime}, n}, & l=1,2,3, \ldots
\end{array}, \Lambda_{m, \tau, \xi}^{4, n}= \begin{cases}\frac{f_{m, 0, \tau-m}^{H^{\prime}, n}}{Z_{0}(0)}, & \xi=0 \\
\frac{f_{m, \xi, \tau-m}^{K^{\prime}, n}}{Z_{\xi}(0)}, & \xi=1,2,3, \ldots\end{cases} \right. \\
T_{m, \tau, l}^{\prime n, j}=\left\{\begin{array}{ll}
\frac{1}{Z_{0}(0)} \sum_{m^{\prime}=-\infty}^{\infty}(-1)^{m^{\prime}} H_{m-m^{\prime}}\left(k_{0} R_{j n}\right) f_{m^{\prime}, 0, \tau-m^{\prime}}^{J^{\prime}, n} \mathrm{e}^{\mathrm{i}\left(m \alpha_{j n}-m^{\prime} \alpha_{n j}\right)}, & l=0 \\
\frac{1}{Z_{l}(0)} \sum_{m^{\prime}=-\infty}^{\infty} K_{m-m^{\prime}}\left(k_{l} R_{j n}\right) f_{m^{\prime}, l, \tau-m^{\prime}}^{I^{\prime}, n} \mathrm{e}^{\mathrm{i}\left(m \alpha_{j n}-m^{\prime} \alpha_{n j}\right)}, & l=1,2,3, \ldots
\end{array} .\right.
\end{gathered}
$$

Since the expression for $f_{\tau, \xi}^{(2), n, i}$ is rather complicated, it is given in Appendix A.

Eqs. (26) and (32) can be seen as a linear algebraic system for the unknown coefficients $A_{m, l}^{n, i, j}$ and $B_{m, l}^{n, i, j}$, which can be solved after truncation. We truncate $(2 M+1)$ terms $(m=-M, \ldots, 0, \ldots, M)$ and $(L+1)$ terms $(l=0,1, \ldots, L)$ in Eqs. (13) and (21), and take $(\tau=-M, \ldots, 0, \ldots, M)$ and $(\xi=0,1, \ldots, L)$ in Eqs. (26) and (32), with the result that a linear algebraic system of $2 N(2 M+1)(L+1)$ order is obtained with the same number of unknown coefficients, which can be solved easily. In the semianalytic computations below, $M=15$ and $L=8$ are found to yield accurate results.

\section{Hydrodynamic coefficients}

The radiation force/moment exerted on Cylinder $n$ ' in Mode $i$ ' due to a unit-amplitude velocity oscillation of Cylinder $n$ oscillating in Mode $i$ is $\operatorname{Re}\left[F_{n, i}^{n^{\prime}, i^{\prime}} \mathrm{e}^{-\mathrm{i} \omega t}\right]$, in which $F_{n, i}^{n^{\prime}, i^{\prime}}$ is the complex amplitude of the force/moment. Since the oscillation of cylinders is harmonic, $F_{n, i}^{n^{\prime}, i^{\prime}}$ can be generally written in terms of hydrodynamic coefficients as:

$$
F_{n, i}^{n^{\prime}, i^{\prime}}=-\mathrm{i} \omega \rho \int_{S_{n^{\prime}}} \Phi_{\mathrm{R}}^{n, i} n_{i^{\prime}} \mathrm{d} s=\mathrm{i} \omega a_{n, i}^{n^{\prime}, i^{\prime}}-c_{n, i}^{n^{\prime}, i^{\prime}},
$$

where the hydrodynamic coefficients $a_{n, i}^{n^{\prime}, i^{\prime}}$ and $c_{n, i}^{n^{\prime}, i^{\prime}}$ are the so-called added-mass and radiation damping coefficients, respectively; $S_{n}$ ' is the wetted surface of Cylinder $n^{\prime} ; n_{i}$ ' is the generalized normal, with $n_{1}=n_{x}, n_{2}=n_{y} \quad n_{3}=n_{z}, n_{4}=-\left(z-z_{n}\right) n_{y}+\left(y-y_{n}\right) n_{z}, n_{5}=\left(z-z_{n}\right) n_{x}-\left(x-x_{n}\right) n_{z}, n_{6}=-\left(y-y_{n}\right) n_{x}+\left(x-x_{n}\right) n_{y}$; and $\vec{n}=n_{x} \vec{i}+n_{y} \vec{j}+n_{z} \vec{k}$ is the normal unit vector at the cylinder surface considered, Eq. (2), which may be written in the $O x y z$ system as

$$
\vec{n}=\frac{1}{\sqrt{1+\left(\frac{1}{r_{n}} \frac{\partial S_{n}}{\partial \theta_{n}}\right)^{2}}}\left(\left(\cos \theta_{n}-\frac{1}{r_{n}} \frac{\partial S_{n}}{\partial \theta_{n}} \sin \theta_{n}\right) \vec{i}+\left(\sin \theta_{n}+\frac{1}{r_{n}} \frac{\partial S_{n}}{\partial \theta_{n}} \cos \theta_{n}\right) \vec{j}+0 \vec{k}\right),
$$

in which $(\vec{i}, \vec{j}, \vec{k})$ are the unit basis vectors in the $O x y z$ system.

\section{Results and discussion}

\subsection{Model validation}

The example used for validation of the present semi-analytic model is two caissons deployed in parallel (Fig. 2), representative of mobile offshore bases (Lamas-Pardo et al., 2015) and offshore floating bridges (Eidem, 2017). 


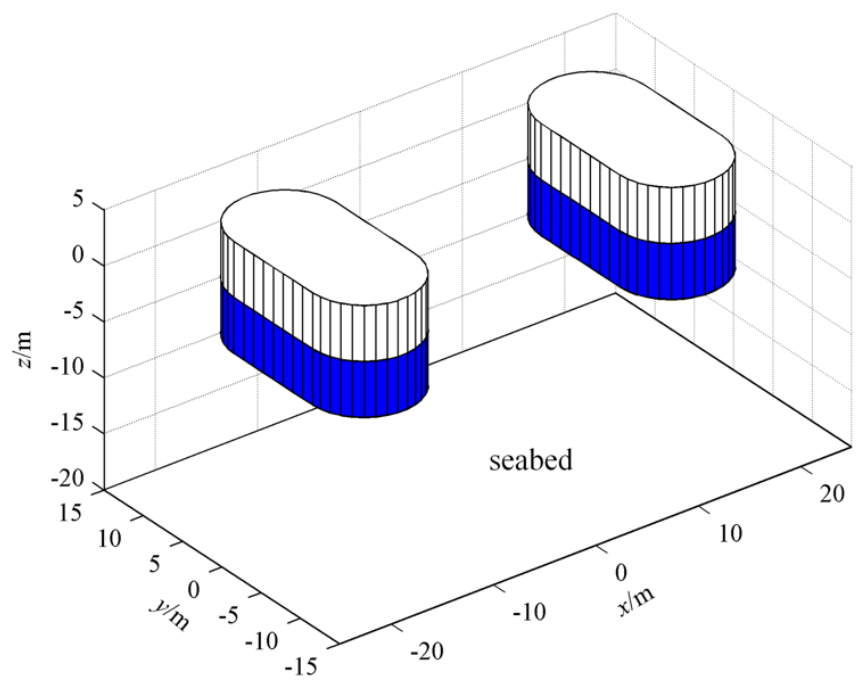

Fig. 2. Case study for validation: two parallel caissons.

In the present case study, the caissons have the same dimensions. The cross section of each caisson consists of a central rectangle of $10 \times 10 \mathrm{~m}$ and two external half circles with a diameter of $10 \mathrm{~m}$. Hence, the functions $S_{n}$ and $\partial S_{n} / \partial \theta_{n}$ of the cross section for each caisson in its cylindrical coordinate system can be written as

$$
S_{n}=r_{n}-\left\{\begin{array}{ll}
\frac{5}{\cos \theta_{n}}, & \left|\theta_{n}\right| \leq \frac{\pi}{4} \\
\frac{-5}{\cos \theta_{n}}, & \left|\theta_{n}-\pi\right| \leq \frac{\pi}{4},
\end{array} \quad \frac{\partial S_{n}}{\partial \theta_{n}}=\left\{\begin{array}{ll}
\frac{-5 \tan \theta_{n}}{\cos \theta_{n}}, & \left|\theta_{n}\right| \leq \frac{\pi}{4} \\
10\left|\sin \theta_{n}\right|, & \text { others } \\
\frac{5 \tan \theta_{n}}{\cos \theta_{n}}, & \left|\theta_{n}-\pi\right| \leq \frac{\pi}{4} \\
-10 \cos \theta_{n}, & \frac{\pi}{4} \leq \theta_{n} \leq \frac{3 \pi}{4} \\
10 \cos \theta_{n}, & -\frac{3 \pi}{4} \leq \theta_{n} \leq-\frac{\pi}{4}
\end{array} .\right.\right.
$$

The spacing between the caissons is $e=20 \mathrm{~m}$. The immersed depth of each caisson and the water depth are $d_{n}=5 \mathrm{~m}$ and $h=20 \mathrm{~m}$, respectively.

The results may be normalized by means of the nondimensional hydrodynamic parameters:

$$
\bar{a}_{n, i}^{n^{\prime}, i^{\prime}}=\frac{a_{n, i}^{n^{\prime}, i^{\prime}}}{\rho h^{j}}, \quad \bar{c}_{n, i}^{n^{\prime}, i^{\prime}}=\frac{c_{n, i}^{n^{\prime}, i^{\prime}}}{\omega \rho h^{j}},
$$

where $j=3$ for $i=1,2,3$ and $i^{\prime}=1,2,3 ; j=4$ for $i=1,2,3$ and $i^{\prime}=4,5,6 ; j=4$ also applies to $i=4,5,6$ and $i=1,2,3$; whereas $j=5$ for $i=4,5,6$ and $i^{\prime}=4,5,6$.

To validate the present semi-analytic model, the hydrodynamic coefficients of the case study (Fig. 2) are evaluated by using both the present semi-analytic model and a BEM-based numerical software, ANSYS-AQWA (ANSYS-AQWA, 2011). Both the semi-analytic results and the numerical results of the frequency responses of the added-mass and radiation damping coefficients for $e / h=1.0$ are plotted in Figs. 3 and 4, in which the frequency is normalized as $k h$ (hereafter, $k$ is adopted as a simplified form of $k_{0}$ ). Figs. $3 \mathrm{a}$ and $4 \mathrm{a}$ illustrate the hydrodynamic coefficients of Caisson 1 regarding the translational modes of oscillation, i.e., surge, sway and heave. The hydrodynamic coefficients of Caisson 1 regarding the rotational modes, i.e., roll, pitch and yaw, are 
1

2

3

4

5
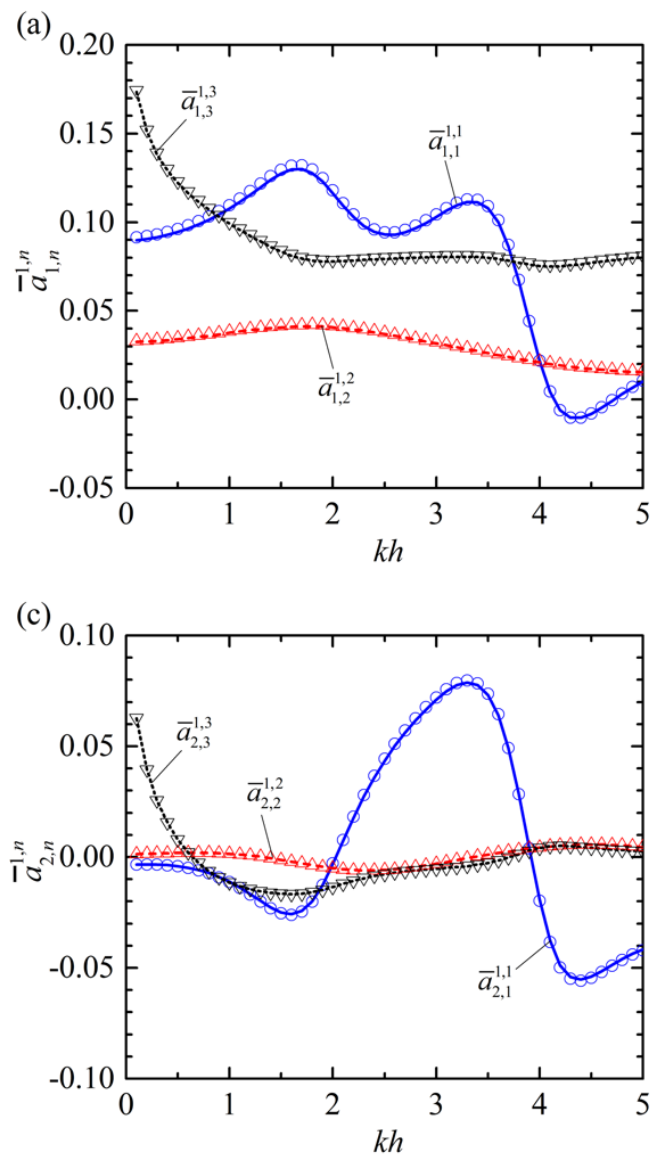

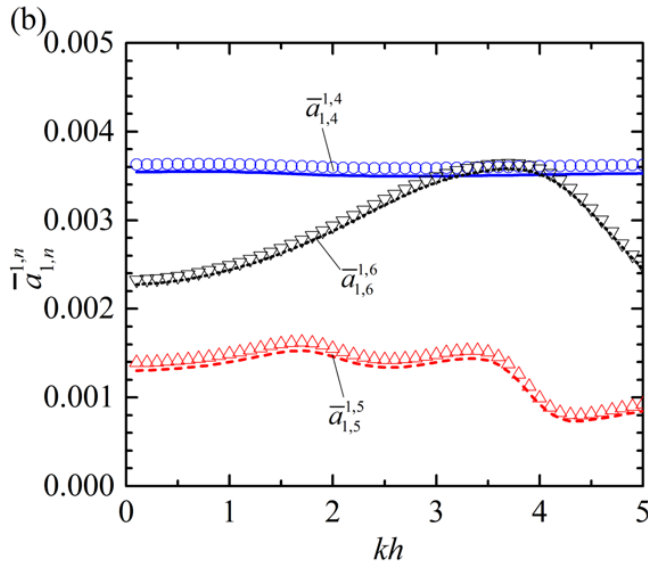

(d)

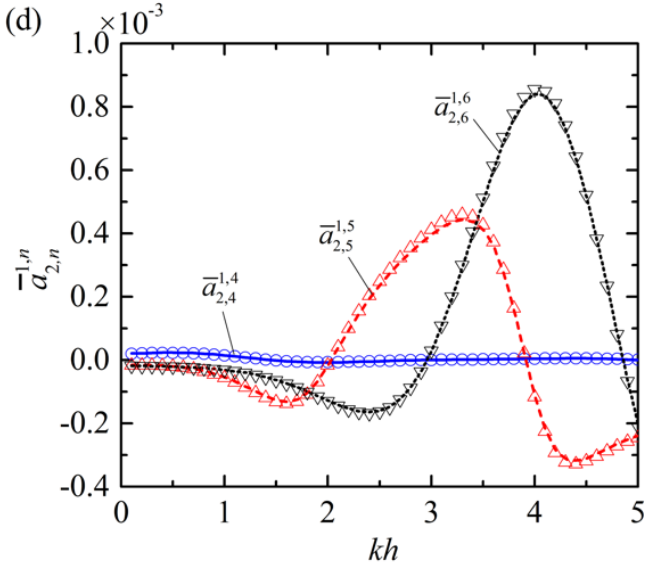

given in Figs. $3 b$ and $4 b$. The results in Figs. $3 c$ and $4 c$ reflect the hydrodynamic interaction between the two caissons in translational modes. Similarly, the hydrodynamic coefficients regarding the hydrodynamic interaction between the two caissons in rotational modes are presented in Figs. 3d and $4 \mathrm{~d}$.

(a) 
(a)

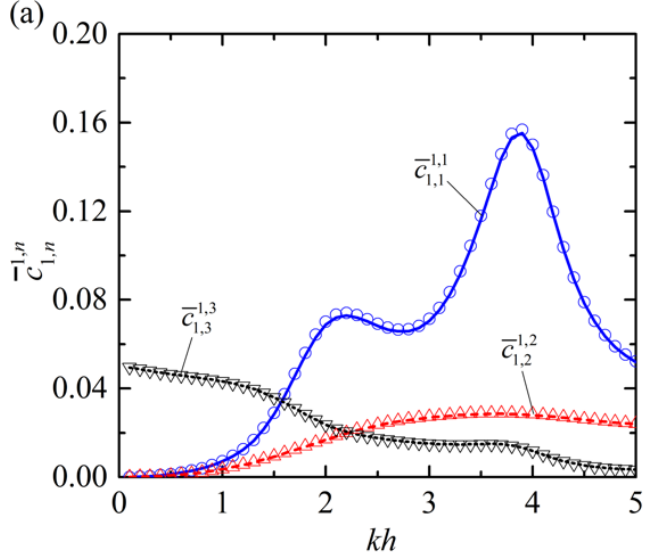

(c)

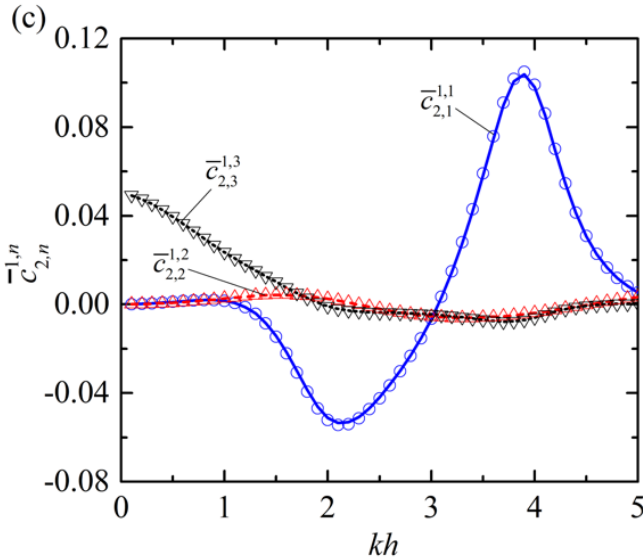

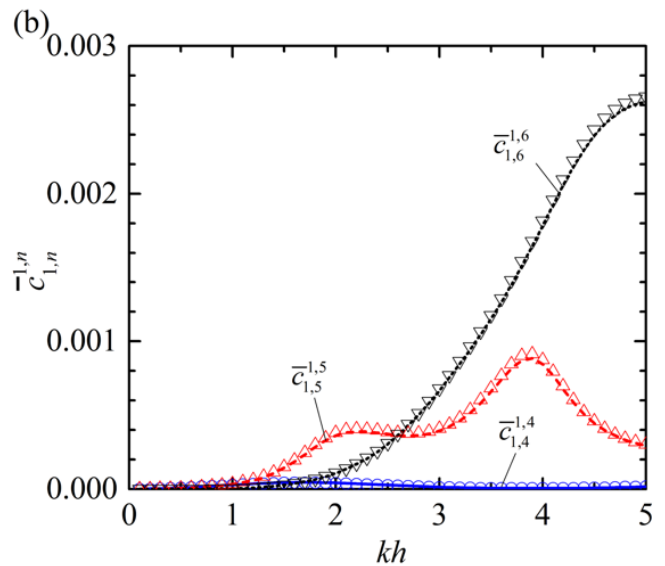

(d)

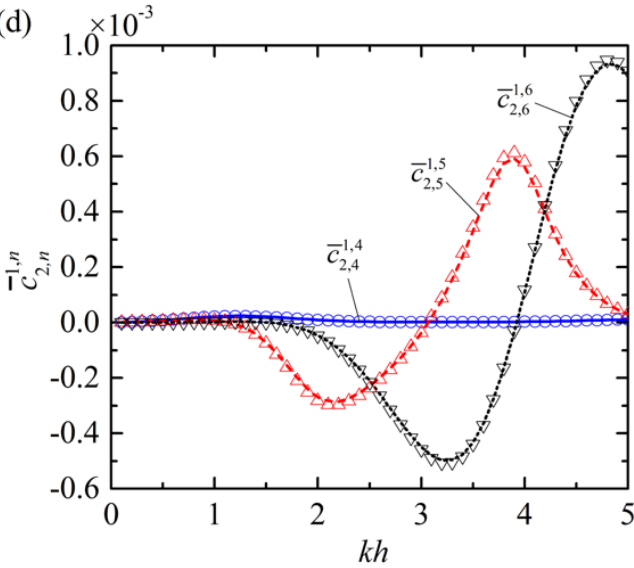

Fig. 4. Radiation damping of the two caissons for $e / h=1.0:$ present semi-analytic results (lines) vs. numerical results (symbols):

(a) $\bar{c}_{1, n}^{1, n}, n=1,2,3$;

(b) $\bar{c}_{1, n}^{1, n}, n=4,5,6$

(c) $\bar{c}_{2, n}^{1, n}, n=1,2,3$;

(d) $\bar{c}_{2, n}^{1, n}$, $n=4,5,6$.

The semi-analytic results and the numerical results in terms of both the added-mass/radiation damping of Caisson 1 and the hydrodynamic coefficients reflecting the hydrodynamic interaction between the two caissons are found to agree with each other. The excellent agreement corroborates the correctness of the semi-analytic model.

In the following subsections, the validated semi-analytic model is employed to study the effect of the spacing distance and the deployment angle on hydrodynamic coefficients.

\subsection{Effect of the spacing between the caissons}

The frequency response of the normalised hydrodynamic coefficients regarding the translational and rotational modes for different spacings, from $e / h=0.5$ to 2.0 , are plotted in Figs. 5 and 6, respectively. Apart from the results of two-caisson cases, hydrodynamic coefficients of an individual caisson floating in isolation are also presented as a comparison. This is equivalent to the two-caisson situation when $e / h$ is large enough to ignore the interaction between the caissons.

Since the two caissons have the same dimensions and are placed in parallel, it follows that $a_{1, n}^{1, n}=a_{2, n}^{2, n}, \quad c_{1, n}^{1, n}=c_{2, n}^{2, n}$. For this reason, the following discussion is concerned with Caisson 1.

Figures 5 and 6 show that $\bar{a}_{1, n}^{1, n}$ and $\bar{c}_{1, n}^{1, n} \quad(n=1,3,5)$ are strongly affected by the spacing between the caissons. The curves representing the frequency responses of these hydrodynamic coefficients oscillate around the curve of the isolated caisson case. This is due to the effects of the hydrodynamic interaction for different $k h$. When Caisson 1 oscillates, apart from the waves radiated 
from the caisson itself, Caisson 1 is also subjected to the waves reflected from Caisson 2, i.e., the radiation force can be divided into two components: the waves radiated from Caisson 1 and those reflected from Caisson 2. The frequency of the reflected waves will change with the oscillating frequency of Caisson 1. Consequently, the propagation time of the reflected waves through the spacing between the two caissons will change, and the hydrodynamic force acting on Caisson 1 due to the reflected waves will change as well in terms of both amplitude and phase. When the two components of the radiation force induced by radiated and reflected waves are in close phase, the hydrodynamic coefficients will be strengthened; whereas if they are out of phase, the hydrodynamic coefficients will be weakened. Take $\bar{a}_{1,1}^{1,1}$ of the two caissons case with $e / h=0.5$ as an example, the peak value of $\bar{a}_{1,1}^{1,1}$ for such case reaches 0.157 at $k h=2.1$, where $\bar{a}_{1,1}^{1,1}$ for the isolated caisson case is only 0.126 , i.e., for $k h=2.1, \quad \bar{a}_{1,1}^{1,1}$ of the two caissons case with $e / h=0.5$ is 0.25 times larger than that of the isolated caisson. The smallest value of $\bar{a}_{1,1}^{1,1}$ of the two caissons case with $e / h=0.5$ occurs at $k h=3.4$, being 0.023 , which is 0.65 times smaller than that $(0.065)$ when the caissons are isolated. It can be learned from Fig. $5 \mathrm{~b}$ that the peak value of $\bar{c}_{1,1}^{1,1}$ with $e / h=0.5$ occurs at $k h=2.6$, which is obviously different from that where the peak of $\bar{a}_{1,1}^{1,1}-k h$ happens.

For the two caissons with a larger value of $e$ and $k h$ ranging from 0 to 5.0, the hydrodynamic coefficient curve of the two caissons oscillates around that of the isolated caisson more frequently, and the oscillating amplitude decreases. This can also be explained from wave reflection from Caisson 2. A larger value of $e$ means a larger propagating distance of the reflected waves, thus for the same variation of $k h$, the phase of the radiation force component induced by the reflected waves changes more, i.e., the strengthening and weakening effects of the hydrodynamic interaction switch more frequently. As $e$ increases, the reflected waves reaching Caisson 1 decrease due to the conservation of wave energy, resulting in weaker hydrodynamic interaction, i.e., smaller oscillating amplitude. Note that for $e / h=1.0$ and 1.5 , negative values of $\bar{a}_{1,1}^{1,1}$ occur around $k h=4.4$ and 5.0, respectively, as a result of the hydrodynamic interaction.

The remaining hydrodynamic coefficients, i.e., $\bar{a}_{1, n}^{1, n}$ and $\bar{c}_{1, n}^{1, n} \quad(n=2,4,6)$, are found to be rather insensitive to the spacing between the caissons, especially $\bar{a}_{1,4}^{1,4}$ (Figs. 5 and 6). 

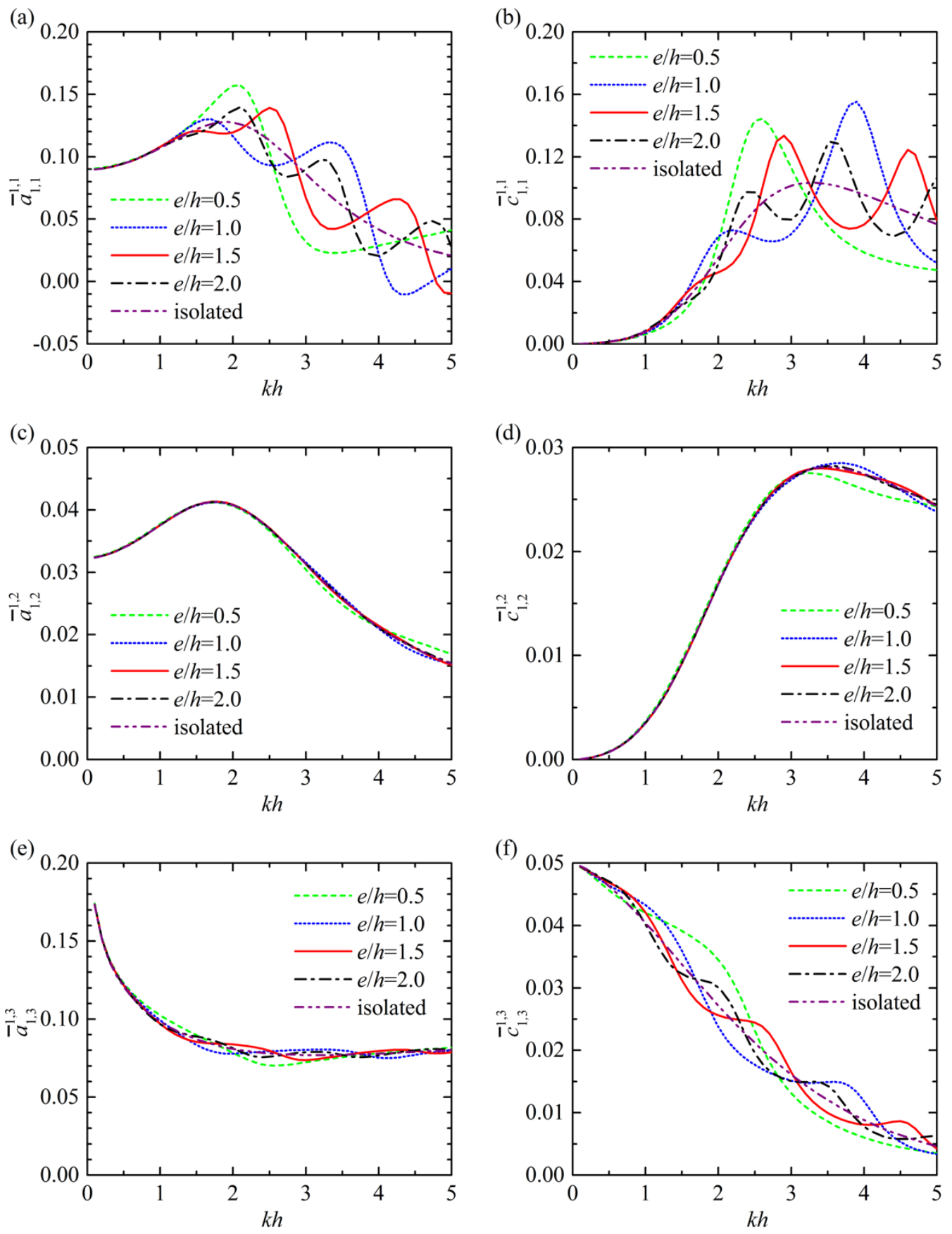

2 Fig. 5. Normalised hydrodynamic coeffieicnts regarding translational modes vs. $k h$ for four spacings:

$3 \quad e / h=0.5,1.0,1.5$ and 2.0, together with the isolated caisson case: (a) $\bar{a}_{1,1}^{1,1}$; (b) $\bar{c}_{1,1}^{1,1}$; (c) $\bar{a}_{1,2}^{1,2}$; (d)

$4 \quad \bar{c}_{1,2}^{1,2} ;(\mathrm{e}) \bar{a}_{1,3}^{1,3} ;(\mathrm{f}) \bar{c}_{1,3}^{1,3}$. 
(a)
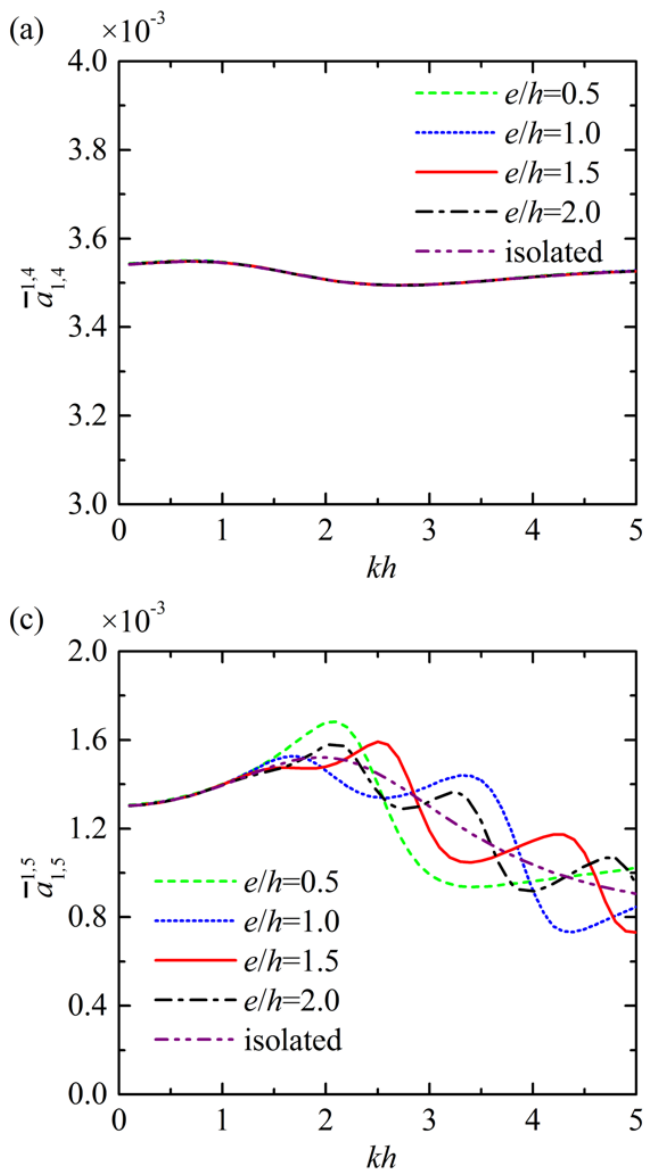

(e)

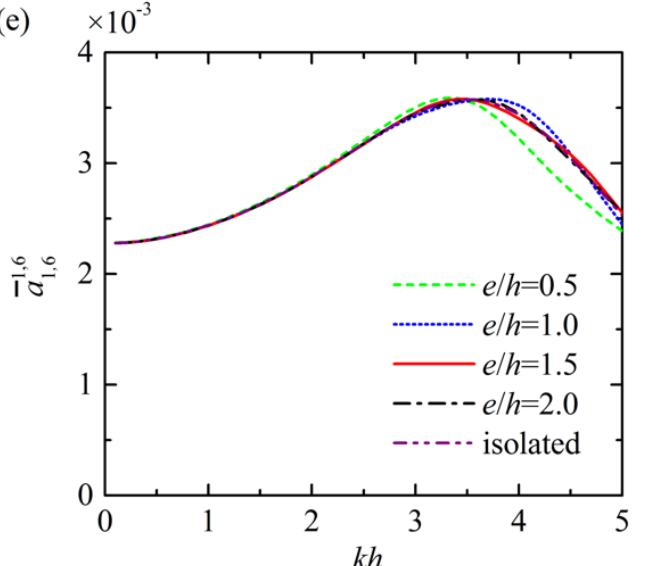

(b)

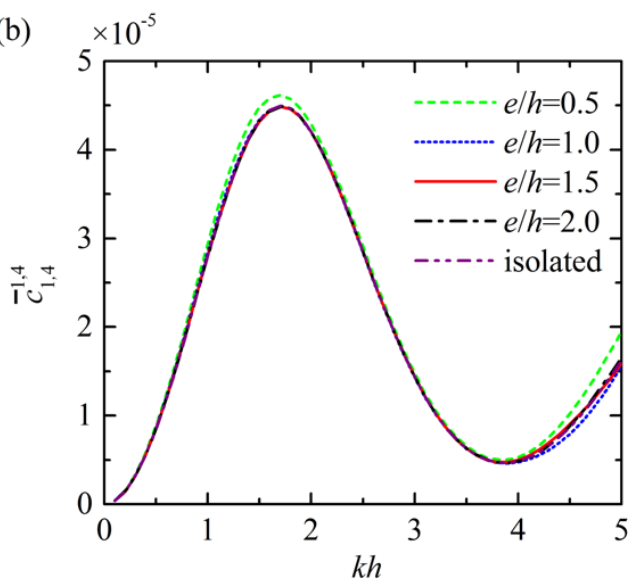

(d)

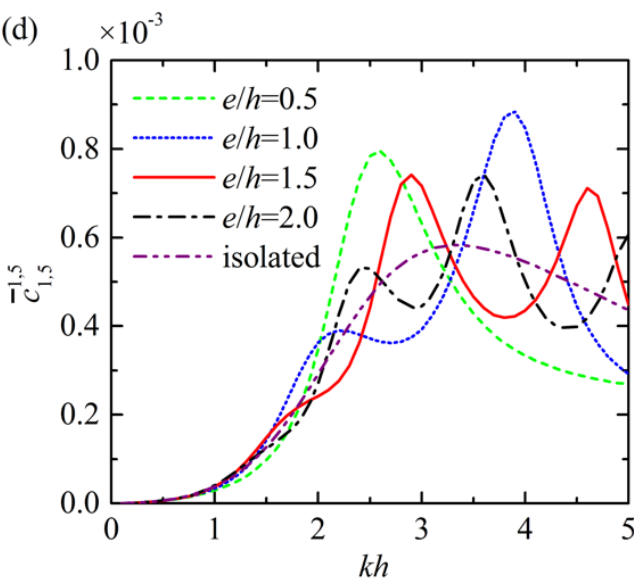

(f)

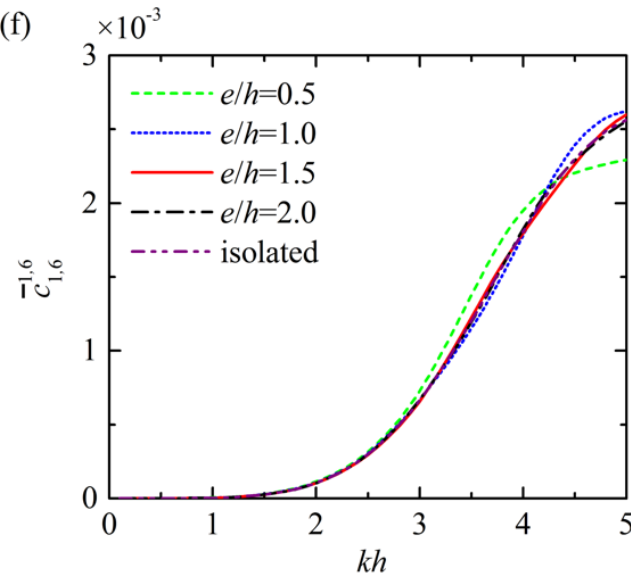

Fig. 6. Normalised hydrodynamic coeffieicnts regarding rotational modes vs $k h$ for four spacings: $e / h=0.5,1.0,1.5$ and 2.0, together with the isolated caisson case: (a) $\bar{a}_{1,4}^{1,4} ;$ (b) $\bar{c}_{1,4}^{1,4} ;$ (c) $\bar{a}_{1,5}^{1,5}$; (d) $\bar{c}_{1,5}^{1,5} ;$ (e) $\bar{a}_{1,6}^{1,6} ;(\mathrm{f}) \bar{c}_{1,6}^{1,6}$.

\subsection{Effect of the deployment angle}

During the construction process of mobile offshore bases and the installation of the floating foundations in floating bridges, one caisson could be dragged around another. Hence, the wave radiation problem of the caissons in different configurations deserves consideration, especially when they are in proximity to each other. This subsection deals with two caissons with the same dimensions as in the previous section in different configurations, i.e., with variant values of the 
deployment angle $\alpha$ (Fig.7).

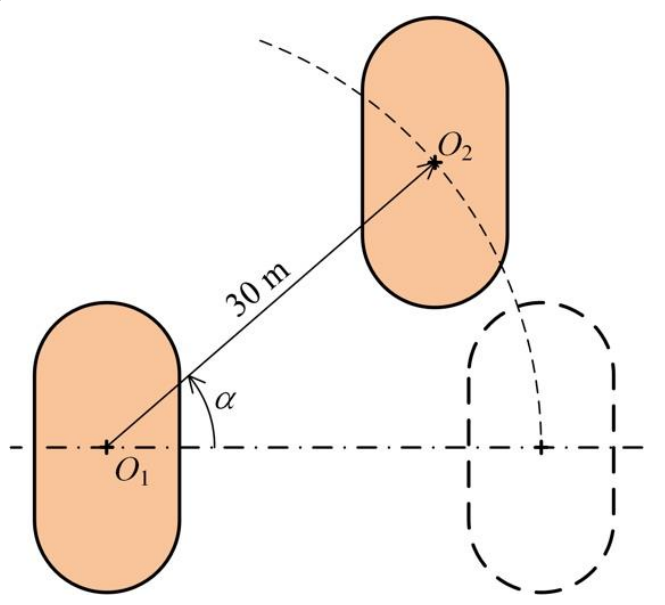

Fig. 7. Definition sketch of the deployment angle

Here the radiation problems from five cases with $\alpha=0, \pi / 6, \pi / 4, \pi / 3$, and $\pi / 2$ are analytically solved. Results of the hydrodynamic coefficients for different cases and their comparison are given in Figs. 8 and 9.

As $\alpha$ increases from 0 to $\pi / 2$, as shown in Figs. $8 \mathrm{a}$ and $8 \mathrm{~b}$, the changing trend of $\bar{a}_{1,1}^{1,1} / \bar{c}_{1,1}^{1,1}$ for different $k h$ can be dramatically different and even opposite. For $2.0<k h<2.6$, the larger the $\alpha$, the larger the $\bar{a}_{1,1}^{1,1}$. Whereas as $\alpha$ increases from 0 to $\pi / 2$, the value of $\bar{a}_{1,1}^{1,1}$ decreases at $k h=1.5$ and 3.5. In Fig. 8b, opposite effects of $\alpha$ on $\bar{c}_{1,1}^{1,1}$ are observed for $1.6<k h<2.1$ and $2.6<k h<3.3$.

Figure $8 \mathrm{c}$ illustrates that as $\alpha$ increases from 0 to $\pi / 2$, the peak value of $\bar{a}_{1,2}^{1,2}$ turns larger and larger, and the corresponding $k h$ is always around 1.7. For $k h$ ranging from 2.8 to $4.1, \bar{a}_{1,2}^{1,2}$ for $\alpha=\pi / 2$ is always smaller than in the other four cases. The frequency response curves of $\bar{c}_{1,2}^{1,2}$ as presented in Fig.8d show obvious fluctuations. The peak value of $\bar{c}_{1,2}^{1,2}$ for different $\alpha$ generally occurs at different wave conditions, e.g., the $k h$ corresponding to the peak of $\bar{c}_{1,2}^{1,2}-k h$ are $3.7,4.1$, 4.4 and 3.0, respectively, for $\alpha=0, \pi / 6, \pi / 4$ and $\pi / 2$.

The effect of $\alpha$ on $\bar{a}_{1,3}^{1,3}$ is found to be rather limited for $0<k h<5.0$ (Fig. 8e). Although for most wave frequencies, $\bar{c}_{1,3}^{1,3}$ changes more or less with the change of $\alpha$, for some specified frequencies, e.g., $k h=1.5$ and 3.0, $\bar{c}_{1,3}^{1,3}$ is found independent of $\alpha$.

The results of $\bar{a}_{1,4}^{1,4}$ (Fig. 9a) show that there is almost no effect of $\alpha$ on $\bar{a}_{1,4}^{1,4}$ regardless of the oscillating frequency of the caisson. The influence of $\alpha$ on $\bar{c}_{1,4}^{1,4}$ mainly appears at $1.6<k h<2.5$ and $k h>4.2$, for which $\bar{c}_{1,4}^{1,4}$ with $\alpha=\pi / 2$ is always the largest one among the five cases (Fig.9b).

The curves of $\bar{a}_{1,5}^{1,5}$ and $\bar{c}_{1,5}^{1,5} \quad$ vs. $k h$ (Figs. $9 \mathrm{c}$ and $9 \mathrm{~d}$ ) are found to be similar to those of $\bar{a}_{1,1}^{1,1}$ and $\bar{c}_{1,1}^{1,1}$ (see Figs. 8a and 8b). For all the five cases with different values of $\alpha$ and $0<k h<5.0$, as given in Fig. 9e, the maximum value of $\bar{a}_{1,6}^{1,6}$ is $3.7 \times 10^{-3}$, occurring at $k h=4.2$ in the case of $\alpha=\pi / 6$. $\bar{c}_{1,6}^{1,6}$ in the same case is also observed to be larger than in the rest of the cases for $4.4<k h<5.0$ (see Fig. 9f). 

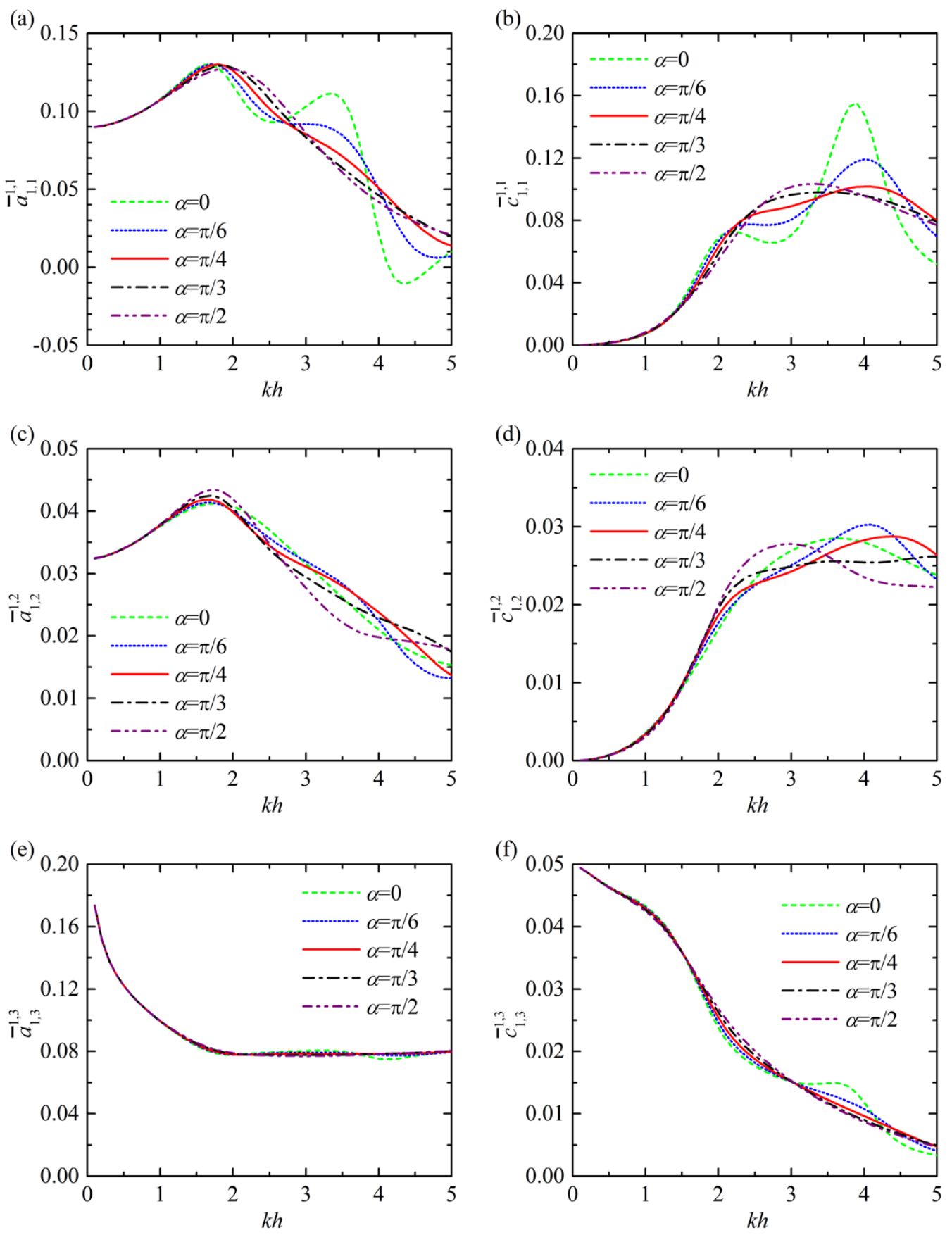

2 Fig. 8. Normalised hydrodynamic coefficients regarding translational modes vs. $k h$ for different

3 deployment angle $\alpha=0, \pi / 6, \pi / 4, \pi / 3$ and $\pi / 2$ : (a) $\bar{a}_{1,1}^{1,1}$; (b) $\bar{c}_{1,1}^{1,1}$; (c) $\bar{a}_{1,2}^{1,2}$; (d) $\bar{c}_{1,2}^{1,2}$;(e) $\bar{a}_{1,3}^{1,3}$;(f) $4 \quad \bar{c}_{1,3}^{1,3}$. 
(a)
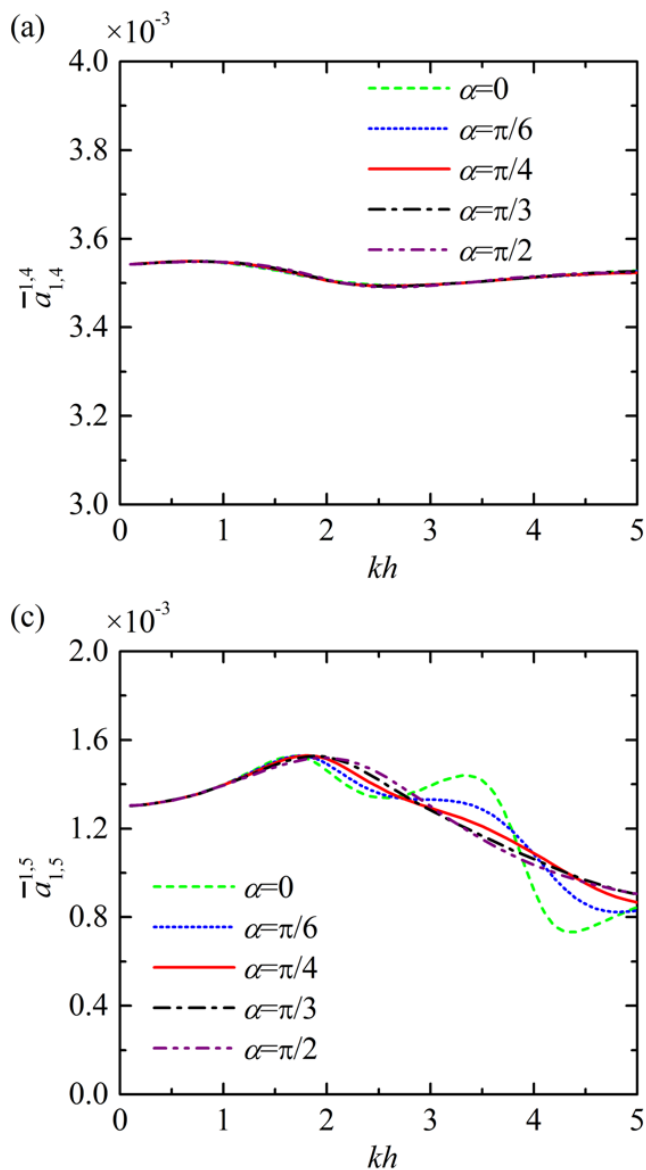

(e) $\times 10^{-3}$

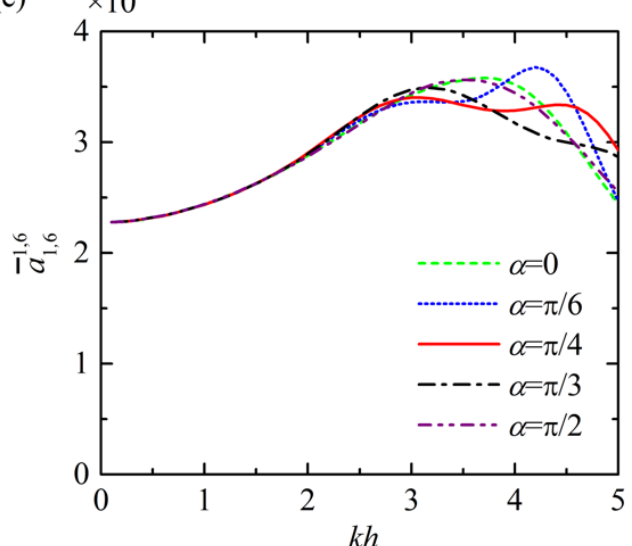

(b)

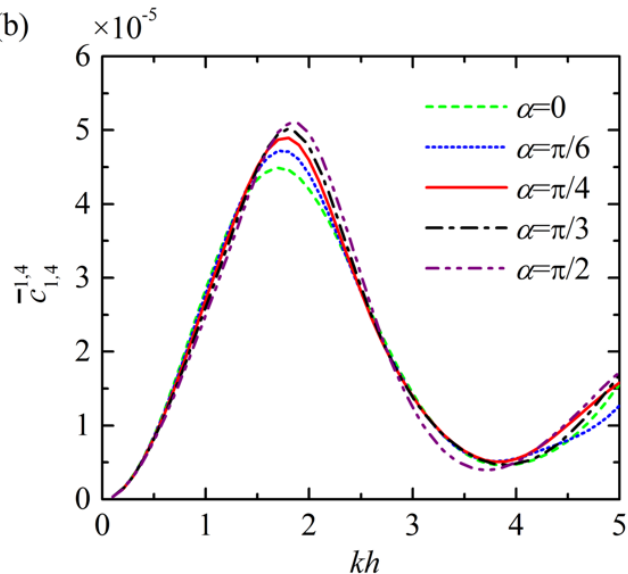

(d)

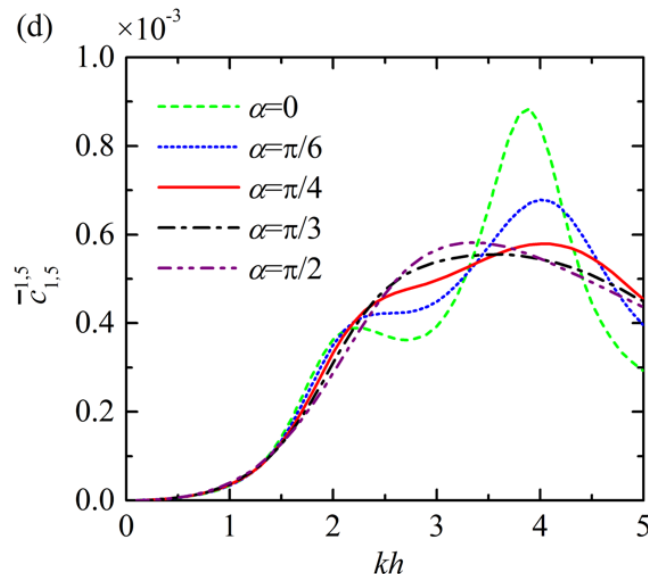

(f)

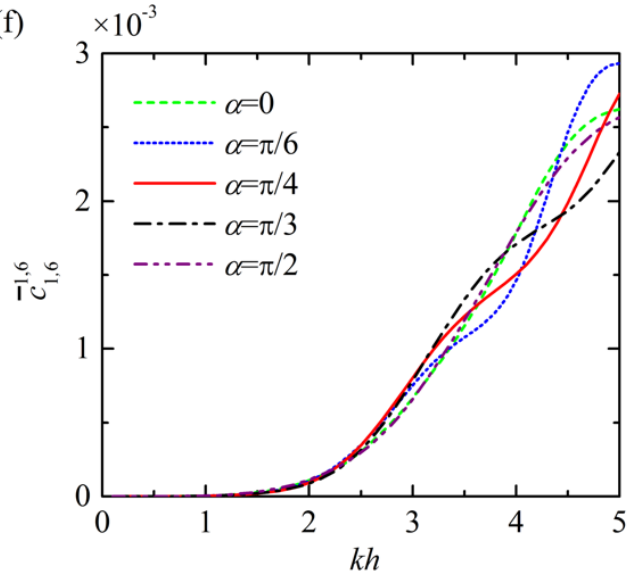

Fig. 9. Normalised hydrodynamic coefficients regarding rotational modes vs $k h$ for different deployment angle $\alpha=0, \pi / 6, \pi / 4, \pi / 3$ and $\pi / 2$ : (a) $\bar{a}_{1,4}^{1,4}$; (b) $\bar{c}_{1,4}^{1,4}$; (c) $\bar{a}_{1,5}^{1,5}$; (d) $\bar{c}_{1,5}^{1,5}$;(e) $\bar{a}_{1,6}^{1,6}$;(f) $\bar{c}_{1,6}^{1,6}$.

\section{Conclusions}

The present research focused on wave radiation from multiple truncated cylinders with arbitrary cross sections. A semi-analytic model was developed to investigate the radiation problem based on linear potential theory. The Fourier-series expansion method combined with the eigenfunction expansion matching method was applied to solve the unknown coefficients in the expressions of the radiated spatial potentials.

This semi-analytic model was validated by comparison with a BEM-based numerical model in 
a case study: two caissons with the same dimensions deployed side by side. Excellent agreement was achieved between the semi-analytic and the numerical results, demonstrating that the present semi-analytic model can successfully solve the problem of wave radiation from multiple floating cylinders of arbitrary cross sections. Upon validation, the semi-analytic model was applied to investigate the effect of the spacing and the deployment angle on the hydrodynamic coefficients (added-mass and wave radiation damping) of the cassisons.

It is found that the hydrodynamic interaction between the caissons can affect the coefficients (relative to the isolated caisson case) depending on the frequency. For this reason, the curves representing the frequency responses of $\bar{a}_{1, n}^{1, n}$ and $\bar{c}_{1, n}^{1, n} \quad(n=1,3,5)$ oscillate around the curve of the isolated caisson case. As the spacing between the two caissons increases, the alternation between strengthening and weakening effects of the hydrodynamic interaction occurs more often for the same interval of $k h$, but the effects themselves gradually wane.

Five configurations of the two caissons with the deployment angle $\alpha$ ranging from 0 to $\pi / 2$ are examined. As $\alpha$ increases from 0 to $\pi / 2$, the trend of $\bar{a}_{1,1}^{1,1} / \bar{c}_{1,1}^{1,1}$ for different frequencies can be dramatically different, and even opposite. Although for most wave frequencies $\bar{c}_{1,3}^{1,3}$ changes more or less with $\alpha$, for certain frequencies, e.g., $k h=1.5$ and 3.0, $\bar{c}_{1,3}^{1,3}$ is found to be independent of $\alpha$. The influence of $\alpha$ on $\bar{c}_{1,4}^{1,4}$ is apparent mainly for $1.6<k h<2.5$ and for $k h>4.2$, for which $\bar{c}_{1,4}^{1,4}$ with $\alpha=\pi / 2$ is always the largest of the five cases.

In sum, the semi-analytic model presented in this paper can be employed to assess the hydrodynamic coefficients of multiple truncated cylinders under small amplitude waves. Together with the semi-analytic model for solving wave diffraction by an array of truncated cylinders with arbitrary cross sections, which will be reported elsewhere, the method can be employed to evaluate the response of many offshore structures, e.g., mobile offshore bases, offshore floating bridges, wave farms and floating offshore wind farms.

\section{Acknowledgements}

This research was supported by China Postdoctoral Science Foundation (Grant No. 2016M601041, 2017T100085), National Natural Science Foundation of China (51679124, 51879144) and Intelligent Community Energy (ICE), INTERREG V FCE, European Commission (Contract No. 5025). The first author wishes to thank Dr. Jiabin Liu from Harbin Institute Technology for valuable discussions. 


$$
f_{\tau, \xi}^{(2), n, i}=\left\{\begin{array}{l}
I_{n, \xi}^{(3)} f_{\tau}^{1, n}, \quad i=1 \\
I_{n, \xi}^{(3)} f_{\tau}^{2, n}, \quad i=2 \\
-\frac{I_{n, \xi}^{(1)}}{2\left(h-d_{n}\right)} f_{3,0, \tau}^{R, n}, \quad i=3 \\
-\left(I_{n, \xi}^{(4)}-z_{n} I_{n, \xi}^{(3)}\right) f_{\tau}^{2, n}+\frac{I_{n, \xi}^{(2)} f_{\tau}^{2, n}}{2\left(h-d_{n}\right)}-\frac{I_{n, \xi}^{(1)} f_{\tau}^{3, n}}{8\left(h-d_{n}\right)}, \quad i=4 \\
\left(I_{n, \xi}^{(4)}-z_{n} I_{n, \xi}^{(3)}\right) f_{\tau}^{1, n}-\frac{I_{n, \xi}^{(2)} f_{\tau}^{1, n}}{2\left(h-d_{n}\right)}+\frac{I_{n, \xi}^{(1)} f_{\tau}^{4, n}}{8\left(h-d_{n}\right)}, \quad i=5 \\
I_{n, \xi}^{(3)} f_{\tau}^{5, n}, \quad i=6
\end{array}\right.
$$

3 where $f_{\tau}^{1, n}, f_{\tau}^{2, n}, f_{\tau}^{3, n}, f_{\tau}^{4, n}, f_{\tau}^{5, n}$ are the Fourier coefficients at the right hand side of Eqs.

$4 \quad$ (A 2a) (A 2e) below and they can be calculated by using Eq.(25).

5

6

7

8

9

$$
\begin{aligned}
& \left.r_{n}\left(r_{n} \cos \theta_{n}-\frac{\partial S_{n}}{\partial \theta_{n}} \sin \theta_{n}\right)\right|_{S_{n}=0}=\sum_{q=-\infty}^{\infty} f_{q}^{1, n} e^{\mathrm{i} q \theta_{n}}, \\
& \left.r_{n}\left(r_{n} \sin \theta_{n}+\frac{\partial S_{n}}{\partial \theta_{n}} \cos \theta_{n}\right)\right|_{S_{n}=0}=\sum_{q=-\infty}^{\infty} f_{q}^{2, n} e^{\mathrm{i} q \theta_{n}}, \\
& \left.r_{j}^{3}\left(3 r_{j} \sin \theta_{j}+\frac{\partial S_{j}}{\partial \theta_{j}} \cos \theta_{j}\right)\right|_{S_{j}=0}=\sum_{q=-\infty}^{\infty} f_{q}^{3, j} e^{\mathrm{i} q \theta_{j}} \\
& \left.r_{j}^{3}\left(3 r_{j} \cos \theta_{j}-\frac{\partial S_{j}}{\partial \theta_{j}} \sin \theta_{j}\right)\right|_{S_{j}=0}=\sum_{q=-\infty}^{\infty} f_{q}^{4, j} e^{\mathrm{i} q \theta_{j}}, \\
& \left.r_{n}^{2} \frac{\partial S_{n}}{\partial \theta_{n}}\right|_{S_{n}=0}=\sum_{q=-\infty}^{\infty} f_{q}^{5, n} e^{\mathrm{i} q \theta_{n}}
\end{aligned}
$$

10

11
$I_{n, \xi}^{(1)}, I_{n, \xi}^{(2)}, I_{n, \xi}^{(3)}$ and $I_{n, \xi}^{(4)}$ are expressed as

$$
I_{n, \xi}^{(1)}=\int_{-h}^{-d_{n}} \frac{Z_{\xi}(z)}{h} \mathrm{~d} z=\left\{\begin{array}{l}
\frac{N_{0}^{-0.5} \sinh \left[k_{0}\left(h-d_{n}\right)\right]}{k_{0} h}, \quad \xi=0 \\
\frac{N_{\xi}^{-0.5} \sin \left[k_{\xi}\left(h-d_{n}\right)\right]}{k_{\xi} h}, \quad \xi=1,2,3, \ldots
\end{array}\right.
$$




$$
\begin{aligned}
& I_{n, \xi}^{(2)}=\int_{-h}^{-d_{n}} \frac{Z_{\xi}(z)}{h}(z+h)^{2} \mathrm{~d} z \\
& =\left\{\begin{array}{l}
\frac{N_{0}^{-0.5}}{k_{0}^{3} h}\left\{k_{0}^{2}\left(h-d_{n}\right)^{2} \sinh \left[k_{0}\left(h-d_{n}\right)\right]-2 k_{0}\left(h-d_{n}\right) \cosh \left[k_{0}\left(h-d_{n}\right)\right]+2 \sinh \left[k_{0}\left(h-d_{n}\right)\right]\right\}, \quad \xi=0, \\
\frac{N_{\xi}^{-0.5}}{k_{\xi}^{3} h}\left\{k_{\xi}^{2}\left(h-d_{n}\right)^{2} \sin \left[k_{\xi}\left(h-d_{n}\right)\right]+2 k_{\xi}\left(h-d_{n}\right) \cos \left[k_{\xi}\left(h-d_{n}\right)\right]-2 \sin \left[k_{\xi}\left(h-d_{n}\right)\right]\right\}, \quad \xi=1,2,3, \ldots
\end{array},\right. \\
& I_{n, \xi}^{(3)}=\int_{-d_{n}}^{0} \frac{Z_{\xi}(z)}{h} \mathrm{~d} z=\left\{\begin{array}{l}
\frac{N_{0}^{-0.5}\left\{\sinh \left(k_{0} h\right)-\sinh \left[k_{0}\left(h-d_{n}\right)\right]\right\}}{k_{0} h}, \quad \xi=0 \\
\frac{N_{\xi}^{-0.5}\left\{\sin \left(k_{\xi} h\right)-\sin \left[k_{\xi}\left(h-d_{n}\right)\right]\right\}}{k_{\xi} h}, \quad \xi=1,2,3, \ldots
\end{array}\right.
\end{aligned}
$$$$
1
$$

3

4

$I_{n, \xi}^{(4)}=\int_{-d_{n}}^{0} \frac{Z_{\xi}(z)}{h} z \mathrm{~d} z=\left\{\begin{array}{l}\frac{N_{0}^{-0.5}}{k_{0}^{2} h}\left\{k_{0} d_{n} \sinh \left[k_{0}\left(h-d_{n}\right)\right]-\cosh \left(k_{0} h\right)+\cosh \left[k_{0}\left(h-d_{n}\right)\right]\right\}, \quad \xi=0 \\ \frac{N_{\xi}^{-0.5}}{k_{\xi}^{2} h}\left\{k_{\xi} d_{n} \sin \left[k_{\xi}\left(h-d_{n}\right)\right]+\cos \left(k_{\xi} h\right)-\cos \left[k_{\xi}\left(h-d_{n}\right)\right]\right\}, \quad \xi=1,2,3, \ldots\end{array}\right.$

\section{References}

Abramowitz, M., Stegun, I.A., 1964. Handbook of mathematical functions. Government Printing Office, Washington, D.C.

ANSYS-AQWA, 2011. Release. Vol. 14., ANSYS-Inc., Pennsylvania, USA.

Bai, W., Eatock Taylor, R., 2006. Higher-order boundary element simulation of fully nonlinear wave radiation by oscillating vertical cylinders. Applied Ocean Research 28 (4), 247-265.

Bhatta, D.D., 2007. Computation of added mass and damping coefficients due to a heaving cylinder. Journal of Applied Mathematics and Computing 23 (1-2), 127-140.

Bhatta, D.D., Rahman, M., 2003. On scattering and radiation problem for a cylinder in water of finite depth. International Journal of Engineering Science 41 (9), 931-967.

Chatjigeorgiou, I.K., 2013. The hydrodynamics of arrays of truncated elliptical cylinders. European Journal of Mechanics B-Fluids 37, 153-164.

Chatjigeorgiou, I.K., Mavrakos, S.A., 2010. An analytical approach for the solution of the hydrodynamic diffraction by arrays of elliptical cylinders. Applied Ocean Research 32 (2), 242-251.

Drobyshevski, Y., 2004. Hydrodynamic coefficients of a floating, truncated vertical cylinder in shallow water. Ocean Engineering 31 (3-4), 269-304.

Eidem, M.E., 2017. Overview of floating bridge projects in Norway, Proceedings of the ASME 2017 36th International Conference on Ocean, Offshore and Arctic Engineering, Trondheim, Norway.

Jiang, S.C., Gou, Y., Teng, B., 2014. Water wave radiation problem by a submerged cylinder. Journal of Engineering Mechanics 140 (5).

Kagemoto, H., Yue, D.K.P., 1986. Interactions among multiple three-dimensional bodies in water waves: an exact algebraic method. Journal Fluid Mechanics 166, 189-209.

Kim, M.H., 1993. Interaction of waves with N vertical circular cylinders. Journal of Waterway Port Coastal and Ocean Engineering-Asce 119 (6), 671-689. 
Krishnankutty, P., Vendhan, C.P., 1995. Three-dimensional finite element analysis of the diffractionradiation problem of hydrodynamically compact structures. Marine Structures 8 (5), 525-542.

Lamas-Pardo, M., Iglesias, G., Carral, L., 2015. A review of very large floating structures (VLFS) for coastal and offshore uses. Ocean Engineering 109, 677-690.

Linton, C.M., Evans, D.V., 1990. The interaction of waves with arrays of vertical circular cylinders. Journal of Fluid Mechanics 215, 549-569.

Liu, J., Guo, A., Fang, Q., Li, H., Hu, H., Liu, P., 2017. Investigation of linear wave action around a truncated cylinder with non-circular cross section. Journal of Marine Science and Technology.

Newman, J.N., 2005. Efficient hydrodynamic analysis of very large floating structures. Marine Structures $18(2), 169-180$.

Sheng, W., Alcorn, R., Lewis, A., 2015. A new method for radiation forces for floating platforms in waves. Ocean Engineering 105, 43-53.

Siddorn, P., Eatock Taylor, R., 2008. Diffraction and independent radiation by an array of floating cylinders. Ocean Engineering 35 (13), 1289-1303.

Taghipour, R., Perez, T., Moan, T., 2008. Hybrid frequency-time domain models for dynamic response analysis of marine structures. Ocean Engineering 35 (7), 685-705.

Teng, B., Ning, D.Z., Zhang, X.T., 2004. Wave radiation by a uniform cylinder in front of a vertical wall. Ocean Engineering 31 (2), 201-224.

Williams, A.N., Darwiche, M.K., 1990. Wave Radiation by Truncated Elliptical Cylinder. Journal of Waterway Port Coastal and Ocean Engineering-Asce 116 (1), 101-119.

Yeung, R.W., 1981. Added mass and damping of a vertical cylinder in finite-depth waters. Applied Ocean Research 3 (3), 119-133.

Yilmaz, O., Incecik, A., 1998. Analytical solutions of the diffraction problem of a group of truncated vertical cylinders. Ocean Engineering 25 (6), 385-394.

Yu, H., Srivastava, M., 2012. Dynamic response of a floating cylinder subjected to coupled wave and wind loading, Proceedings of the ASME 2012 International Mechanical Engineering Congress and Exposition, Houston Texas, USA, pp. 1731-1742.

Yu, H., Zheng, S., Zhang, Y., Iglesias, G., 2019. Wave radiation from a truncated cylinder of arbitrary cross section. Ocean Engineering 173, 519-530.

Zeng, X.H., Tang, S.Y., 2013. The hydrodynamic interactions of an array of truncated circular cylinders as each cylinder oscillates independently with different prescribed modes. Journal of Hydrodynamics 25 (1), 27-38.

Zhang, S., Williams, A.N., 1996. Wave scattering by submerged elliptical disk. Journal of Waterway Port Coastal and Ocean Engineering-Asce 122 (1), 38-45.

Zheng, S., Zhang, Y., 2015. Wave diffraction from a truncated cylinder in front of a vertical wall. Ocean Engineering 104, 329-343.

Zheng, S., Zhang, Y., 2016. Wave radiation from a truncated cylinder in front of a vertical wall. Ocean Engineering 111, 602-614.

Zheng, S., Zhang, Y., 2018. Theoretical modelling of a new hybrid wave energy converter in regular waves. Renewable Energy 128A, 125-141.

Zheng, S., Zhang, Y., Iglesias, G., 2018. Wave-structure interaction in hybrid wave farms. Journal of Fluids and Structures 83, 386-412. 